\title{
Claude Rochet
}

(1992)

\section{Managez vos associations}

Préface de Kléber Beauvillain

\section{Partie 1}

Un document produit en version numérique par Mme Marcelle Bergeron, bénévole Professeure à la retraite de l'École Dominique-Racine de Chicoutimi, Québec et collaboratrice bénévole Courriel : mailto:mabergeron@videotron.ca

Dans le cadre de la collection : "Les classiques des sciences sociales" dirigée et fondée par Jean-Marie Tremblay, professeur de sociologie au Cégep de Chicoutimi

Site web : http://classiques.uqac.ca/

Une collection développée en collaboration avec la Bibliothèque

Paul-Émile-Boulet de l'Université du Québec à Chicoutimi

Site web: http://bibliotheque.uqac.uquebec.ca/index.htm 
Un document produit en version numérique par Mme Marcelle Bergeron, bénévole, professeure à la retraite de l’École Dominique-Racine de Chicoutimi, Québec.

Courriel : mailto:mabergeron@videotron.ca

Claude Rochet.

Une édition électronique réalisée à partir du texte de Claude Rochet, Managez vos associations; Préface de Kléber Beauvillain Paris: CALMANN-LÉVY, Éditeur, 1992, 289 pp.

Polices de caractères utilisés :

Pour le texte : Times, 12 points.

Pour les citations : Times 10 points.

Pour les notes de bas de page : Times, 10 points.

Édition électronique réalisée avec le traitement de textes Microsoft Word 2003 pour Macintosh.

Mise en page sur papier format

LETTRE (US letter), 8.5'’ x 11’’)

Édition complétée le 27 juin, 2006 à Chicoutimi, Québec. 


\section{Claude Rochet}

Managez vos associations

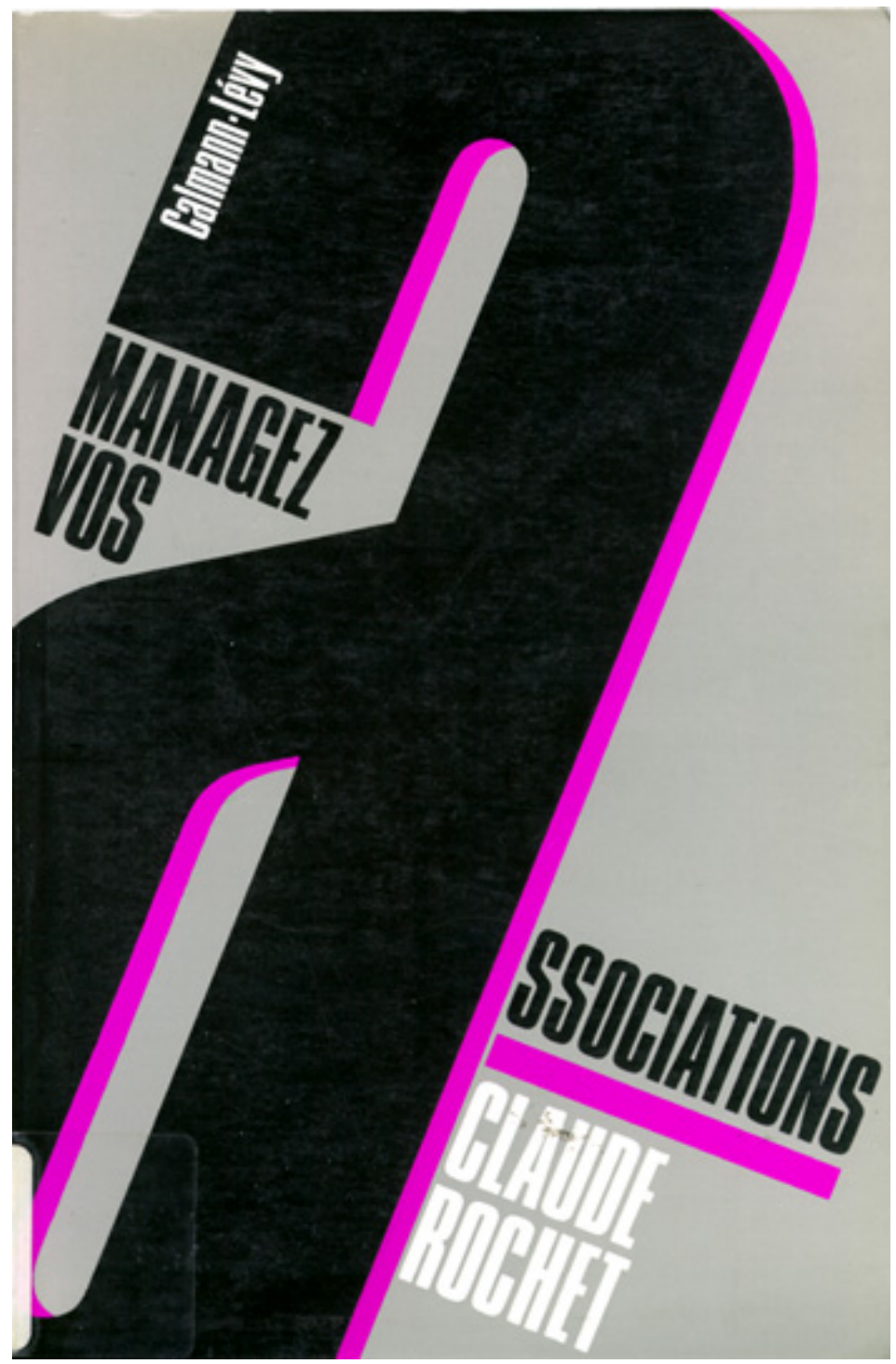




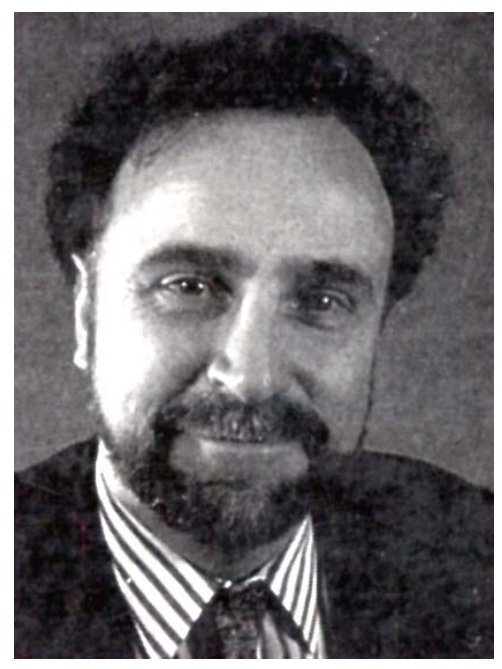

\section{Présentation du livre}

(Texte au verso du livre)

Plus de 600000 associations jouent aujourd'hui en France un rôle irremplaçable pour la dynamique de la société civile. Certaines sont d'énormes organisations, employant plusieurs centaines ou milliers de salariés et évoluant dans un environnement complexe, parfois international. D'autres sont de petites structures assurant des fonctions essentielles à l'échelle locale. Toutes souffrent pourtant d'un véritable déficit d'organisation qui fait obstacle à leur développement et les condamne souvent à vivoter ou à disparaître.

Comment faire pour qu'une association soit efficace, qu'elle soit au service de ses adhérents, que la qualité de ses services soit la meilleure possible ? Comment mobiliser des bénévoles, ne pas se tromper d'objectif, déjouer les pièges d'une croissance mal contrôlée, trouver l'argent nécessaire à son développement, mesurer la qualité de son service, concevoir une politique marketing, bien gérer ses recettes et ses dépenses, fixer les bons prix de ses prestations et négocier sur des bases saines avec les pouvoirs publics ?

À ces questions, une seule réponse: le management qui signifie tout simplement faire tout ce qu'il faut pour que le résultat visé soit atteint. Face aux défis qu'affronte la société française, c'est aujourd'hui plus qu'une méthode, une ardente obligation.

Énarque, ancien cadre dirigeant de Sollac (groupe Usinor Socilor), Claude Rochet dirige aujourd'hui le cabinet Référence Conseil, spécialisé dans la conduite du changement. Il intervient à ce titre auprès de grandes entreprises industrielles et de nombreuses associations.

Klébert BEAUVILLAIN, Président de Hewlett-Packard 


\section{Table des matières}

\section{Préface de Kléber Beauvillain}

Introduction. Pourquoi les associations ont-elles besoin d'être
managées?

Première partie.

L'OUTIL ASSOCIATIF

\section{L'association : un contrat pour entreprendre.}

Un droit longtemps suspect.

Une bombe à retardement.

Une entreprise de cohésion sociale.

La cohésion du système social : le modèle tridimensionnel.

2. Un outil de proposition et d'innovation sociale.

Une entreprise de projet à vocations multiples.

Le mode de production associatif.

\section{Deuxième partie \\ UN PROJET}

\section{De l'idée au projet.}

Bâtir un projet : une offre d'idées face à une demande sociale.

Un besoin, un marché, un projet.

À quoi ça sert ? L'analyse des besoins.

L'étude de marché.

Évaluer et dépasser les contraintes.

\section{Du projet au client.}

Le marketing, ou comment ne plus parler d'argent.

Qu'est-ce que le marketing ?

Organiser l'échange.

Choisir et être choisi.

La mise en œuvre d'une stratégie marketing.

Le marketing : un outil qui peut se raffiner... à l'excès.

La qualité : y a mieux, mais c'est moins cher.

Pas de marketing possible sans qualité.

Comment fabrique-t-on la qualité ? 
Améliorer la qualité.

Les réseaux.

Identifier le jeu des acteurs.

Les moyens.

La communication : faire savoir pour faire faire.

Vie et mort du projet.

\section{Troisième partie \\ DES HOMMES}

\section{Les bénévoles.}

Adhérer à une association, c'est adhérer à un contrat qui devient la loi pour chaque partie.

Première règle: examiner attentivement chaque demande d'adhésion.

Deuxième règle : des membres bien vivants.

Troisième règle : varier les genres et les talents.

Quatrième règle : choisir sans attendre d'être choisi.

Cinquième règle : choisir un bon président.

Acteurs et consommateurs.

Les petites, les moyennes et les grosses.

Le jeu associatif : vive le conflit !

Première règle : tout le monde a le droit de s'exprimer même si c'est pour dire n'importe quoi.

Deuxième règle déconcentrer l'initiative.

Troisième règle respecter le principe de libre contribution des membres.

Quatrième règle : professionnaliser le bénévolat par un contrat gagnant-gagnant.

Organiser le foisonnement.

Le grand jeu : l'assemblée générale.

\section{Les salariés.}

Contrat de travail et contrat d'association.

Première règle : conserver la stratégie, déléguer la gestion.

Deuxième règle: bien répartir les rôles entre exécutif élu et salariés.

Troisième règle : le salarié n'est pas un militant.

Quatrième règle : toute embauche se fait sur profil de poste.

Cinquième règle: jouer le professionnalisme contre le militantisme.

Sixième règle : gérer la sortie. 
Payer mieux pour payer moins.

Première règle : analyser et optimiser l'emploi existant avant de décider une nouvelle embauche.

Deuxième règle : évaluer l'incidence de l'emploi en dépenses et en recettes.

Troisième règle : payer le juste prix.

Quatrième règle : valoriser les résultats.

Jouer gagnant-gagnant.

Première règle : tous gagnants !

Deuxième règle : $1+1>3$.

Troisième règle : établir des mandats clairs pour s'agiter moins et travailler plus.

Quatrième règle : tous PDG !

\section{Quatrième partie}

DES MOYENS

\section{Le capital pu le dilemme de la cigale et de la fourmi.}

\section{Les ressources.}

Vendre pour dépenser : les ressources propres.

Le portefeuille d'activités et les services rendus.

Combien ça coûte ?

Quel prix fixer?

Parrainage et mécénat : l'imagination au pouvoir.

Mécénat, mode d'emploi.

Première règle : faire mécéner avant tout les coûts variables.

Deuxième règle : fidéliser les financements en provenance du mécénat.

Troisième règle : personnaliser les relations avec les mécènes.

Subventions, danger!

Les subventions de fonctionnement: un réel danger pour un avantage douteux.

Comment bien utiliser les subventions.

Première règle: une subvention est un solde obtenu après épuisement de toutes les autres sources de financement.

Deuxième règle: préférer les subventions ponctuelles ou indirectes.

Troisième règle : une subvention est la contrepartie d'un service rendu à la collectivité.

Quatrième règle : la convention doit être un acte de management.

\section{Cinquième partie}




\section{CONDUIRE LE CHANGEMENT}

Le management n'est pas compliqué ; il est complexe.

Un peu de théorie : qu'est-ce qu'un système ?

Gérer la complexité, c'est mettre de la souplesse et de la cohérence dans son moteur.

Comment s'en sortir?

D'où l'on vient et où l'on va.

Première règle : fixer des objectifs clairs et ambitieux.

Deuxième règle : ne pas chercher des solutions avant d'avoir posé le problème.

Troisième règle : mesurer l'écart.

Quatrième règle : banco sur le multiplicateur participatif !

Cinquième règle : payer le ticket d'entrée.

La ressource humaine, principal facteur ou obstacle du changement.

Première règle : le changement marche au mélange.

Deuxième règle : que les dirigeants montrent l'exemple !

Troisième règle : convertir les sceptiques par les pionniers.

Quatrième règle : bâtir une nouvelle règle du jeu.

Planifier la démarche.

Rechercher des résultats progressifs et significatifs.

Mettre en place des structures participatives.

Développer l'expérimentation.

Commencer par la formation, consolider par la formation, boucler par la formation.

Remerciements. 


\section{Préface}

$\underline{\text { Retour à la table des matières }}$

Terre de contraste et de passion, la France, après avoir mythifié l'État, a découvert des lettres de noblesse à l'entreprise. Ce n'était là que justice : créatrice de valeur ajoutée, clé de la compétitivité de la maison France, elle a contribué ces dernières années à une révolution profonde du management désormais basé sur la valorisation de la personne, la créativité et l'initiative individuelle. Face à une complexité réelle et une compétition de plus en plus aiguë, des efforts ont été entrepris, avec des fortunes diverses mais des résultats certains. L'entreprise a un atout sur les autres corps sociaux : si elle ne se lance pas dans l'aventure du management, elle meure !

Ces succès doivent, toutefois, être nuancés. Ils ne doivent pas être l'arbre qui cache la forêt : l'entreprise ne sait ni ne doit tout faire. Il faut être prudent lorsqu'on lui demande - au travers du mécénat — de prendre en charge des actions dans le domaine de la culture ou de l'aide humanitaire. Si cela peut lui apporter beaucoup en ouverture d'esprit et en expérience, ce n'est quand même pas son métier de base. L'entreprise est avant tout au service de ses clients pour lesquels elle développe en permanence son savoir-faire. Ce n'est pas parce qu'elle réussit, tandis que les autres institutions (État, collectivités locales, associations) cherchent leurs marques, qu'il faut lui demander tout et n'importe quoi !

Un pays ne se réduit pas à la somme de ses entreprises. Une entreprise performante ne le sera plus dans une société qui ne l'est pas. L'homme se nourrit d'autres choses que de résultats d'exploitation. Son rêve, sa vision du futur s'épanouissent dans l'entreprise associative pour des objectifs tout aussi louables que dans l'entreprise industrielle ou commerciale.

Nous sommes tributaires de la qualité de notre environnement social, de l'appareil éducatif à la protection de la nature. L'homme ne s'épanouit pas seulement sur son lieu de travail — cela a même été pendant longtemps le contraire ! - mais avant tout dans sa vie sociale. Et cette vie sociale — dont l'association a vocation à être la cheville ouvrière - a tout à gagner au 
concours de cadres hautement qualifiés d'une économie hypercompétitive qui vont venir lui apporter leur enthousiasme et leur savoir-faire.

Entre l'association et l'entreprise, c'est un véritable contrat gagnantgagnant qui peut se construire ! Il y a, en effet, une réelle complémentarité de savoir-faire :

- D'industrie de produits nous passons désormais à une industrie de services. C'est — en jargon d'informaticien - la prééminence du soft sur le hard. Ce service est avant tout immatériel, et tout aussi difficile à évaluer que l'apport d'une action sociale ou culturelle à la collectivité.

- Nous devons tous sortir de la contemplation de notre pré carré pour penser nos projets comme des fonctions utiles au plus grand nombre. Quoi que nous fassions, nous ne devons plus seulement chercher à nous faire plaisir ou à nous mettre en avant, mais viser avant tout à créer de la valeur pour les consommateurs de nos services, nos clients.

- Tous, nous avons à mobiliser les femmes et les hommes de nos organisations qui en sont le principal capital et la ressource fondamentale. Pour dépasser nos corporatismes, nos visions étroites de spécialistes, nous devons apprendre à raisonner pour le bien commun.

L'opposition entre intérêt général et intérêt privé paraît, à cet égard, particulièrement nocive, et c'est le grand mérite de Claude Rochet que de nous proposer d'abattre cette frontière stérile entre le «lucratif » et le «non lucratif ».

Alors «managez vos associations » comme une entreprise ? Surtout pas! Les associations ont leur originalité et leur complexité qui en rendent le pilotage bien plus délicat qu'une entreprise: le client n'est pas toujours le payeur, la valeur produite est plus délicate à mesurer, exiger du professionnalisme de gens qui travaillent bénévolement est un savoir-faire tout particulier. Nous devons donc apprendre à manager nos associations... mieux qu'une entreprise !

Claude Rochet prend, pour la première fois, l'initiative de mettre à la portée de tous les méthodes et outils développés dans les organisations les plus performantes: conception du projet, mobilisation des hommes, qualité, marketing, communication, maîtrise des moyens et pilotage du changement trouvent ici leur déclinaison propre à l'association. Le sérieux s'allie dans cet ouvrage à l'humour et à un esprit décapant. Une opportunité à ne pas manquer !

Kléber BEAUVILLAIN

Président de Hewlett-Packard France 


\section{Introduction}

\section{Pourquoi les associations ont-elles besoin d'être managées?}

$\underline{\text { Retour à la table des matières }}$

T e droit d'association est une liberté publique constitutionnelle qui ouvre Laux citoyens le droit de s'organiser pour «mettre en commun, de façon permanente, leurs connaissances ou leur activité dans un but autre que partager des bénéfices » (loi du $1^{\mathrm{er}}$ juillet 1901). Est association tout ce qui n'est pas autre chose, une société commerciale notamment, et qui peut exister avec un minimum de formalités dès qu'il y a deux personnes décidées à entreprendre un projet commun. L'association est donc une véritable entreprise de projet à vocation multiple.

Le management, c'est penser la gestion en termes de projet, c'est parvenir à asservir l'ensemble des techniques d'organisation à la réalisation d'un objectif. C'est l'art de faire ce qu'il faut pour que le résultat visé soit atteint. Ces notions vont de soi dans les démocraties anglo-saxonnes où la libre initiative des citoyens est à la base de l'organisation sociale ; elles le sont moins en France, pays de centralisation administrative. L'association y est encore peu répandue en comparaison avec les pays de démocratie décentralisée, et elle s'est développée dans le moule culturel de l'administration publique qui repose sur trois principes :

— Recherche de la légitimité dans la conformité à des normes abstraites et intemporelles.

- Primauté du contrôle de la conformité aux normes sur l'efficacité et l'action.

— Méfiance de principe à l'égard de l'initiative individuelle et privée.

L'association se trouve ainsi handicapée de se penser comme un pseudopode ${ }^{1}$ de la puissance publique avec deux conséquences fondamentales qui sont aux antipodes du management :

1 Prolongement tentaculaire. 
- L'association reproduit plus qu'elle n'invente : une grande fédération associative a été jusqu'à copier sa convention collective sur le statut de la fonction publique. Il existe beaucoup de manuels d'excellente qualité de réglementation et de droit des associations, il n'en existait jusqu'à ce jour aucun de management. Que penser d'un chef d'entreprise qui puiserait toute son inspiration dans un manuel de droit des affaires! C'est pourtant dans ce genre de situation que se trouvent les associations.

- Un comportement d'assisté de la puissance publique qui se traduit par une quête permanente et obsessionnelle de subventions, qui deviennent des rentes de situation dont il est hors de question de rechercher l'utilité ou la bonne utilisation.

L'évolution des interventions de la puissance publique a rendu ce modèle obsolète. L'échec de la dernière tentative de relance économique par les finances publiques (la politique Mauroy de 1981 à 1983) a montré les limites de l'initiative unique et centralisée, ainsi que l'importance d'une prise en compte des multiples interactions qui parcourent une société devenue hautement complexe. Dans ce vieux pays de droit régalien où l'initiative publique a toujours été bonne parce que publique, on a découvert qu'un emploi improductif financé sur fonds publics contribuait à l'extension du chômage en pesant sur la compétitivité des emplois productifs. Sous la double pression de la raréfaction des crédits publics et de l'intensité des problèmes sociaux s'est révélé l'intérêt de rechercher des partenaires privés à l'action publique, tant pour des raisons financières que d'adaptation des politiques au terrain et de souplesse de mise en œuvre. On a découvert l'importance des initiatives de la société civile dont l'association est le mode d'organisation le plus élémentaire.

Le paysage associatif français est aujourd'hui très composite, et se répartit sommairement en deux catégories :

- Les associations anciennes qui ont grandi avec les subventions publiques et s'en sont intoxiquées. Elles se trouvent en état de manque - d'idées et d'argent - pour entretenir des structures où l'on trouve plus de salariés que de membres actifs.

- Les associations récentes qui se sont développées - ou rénovées depuis les années soixante-dix, sans rechercher l'onction de la puissance publique et ont su mettre en place une gestion performante adaptée aux ambitions du projet.

Le propos de cet ouvrage est de penser l'association comme un contrat fédérateur de volontés individuelles, émancipé de la tutelle publique, bâti autour de l'ouverture d'esprit et de l'imagination créatrice pour la conception du projet, et du pragmatisme pour sa mise en œuvre.

Le management part du principe qu'il y a une demande sociale à laquelle il faut répondre par une offre de produits élaborés dans un lieu de production 
qu'est l'association. Son objectif est de rechercher la meilleure organisation de l'entreprise associative pour que le produit réponde effectivement à la demande.

Cela suppose que les managers d'associations abandonnent cette position ambiguë, entre des velléités d'indépendance et la vieille conviction que l'action associative n'est pas une fin en soi mais nécessite d'être relayée par l'administration ou l'action politique.

Émanation de la société civile, l'association est le lieu rêvé pour que s'élaborent des circuits courts de production de biens collectifs sans passer par l'administration. L'aide humanitaire, le secours au quart monde, l'entraide des chômeurs sont des initiatives associatives face à des problèmes auxquels la puissance publique n'apportait pas de réponse.

Adopter la logique de l'économie de marché n'est donc pas céder à une quelconque mode passagère mais rechercher une plus grande efficacité dans la production de ces services: meilleure adaptation de l'offre à la demande, adaptation de la structure au projet, et redéfinition des rapports entre les associations et les pouvoirs publics. L'association offre un service à la collectivité qui en reconnaît l'intérêt au travers d'une convention définissant les objectifs communs poursuivis et le niveau de sa contribution.

Peut-être peut-on gérer une association comme une entreprise, sans doute faut-il la gérer mieux qu'une entreprise, car c'est une structure plus complexe :

- La rentabilité ne sera pas seulement financière mais surtout sociale : l'objectif est-il atteint dans les meilleures conditions au regard des moyens mis en œuvre?

— L'association aura à observer certaines règles éthiques qui seront autant de contraintes pesant sur sa gestion.

- L'association gère un double réseau de membres bénévoles et de salariés, ce qui rendra la gestion des ressources humaines particulièrement complexe.

Cet ensemble de contraintes fait du management associatif un domaine encore bien peu exploré. Cet ouvrage propose une méthodologie d'approche en quatre temps :

- La définition du projet.

— La mobilisation des femmes et des hommes pour sa mise en œuvre.

- La mise en place des moyens nécessaires.

- La conduite du changement qui permettra d'adapter l'organisation au projet. 


\title{
Première partie
}

\author{
L'OUTIL ASSOCIATIF
}

L'association : un contrat pour entreprendre

$\underline{\text { Retour à la table des matières }}$

Liberté publique permettant aux citoyens de prendre en main leurs intérêts, le droit d'association est encore peu développé en France. À un premier âge associatif qui a vu les associations se développer dans l'orbite des institutions publiques succède un second âge où le développement de la vie associative s'appuie sur l'initiative de citoyens dont l'entreprise ne pourra plus consommer toute la créativité. L'association, par le développement de ses performances au travers du management, est appelée à devenir une véritable entreprise de cohésion sociale tendant un pont sur le précipice qui tendra à s'élargir entre exclus et travailleurs d'une société de plus en plus performante.

Doint n'est besoin pour entreprendre de se doter de structures juridiques complexes et d'avoir des moyens financiers considérables. Les aurait-on que cela ne dispenserait pas de l'ingrédient essentiel : le projet. Un projet fait intervenir une offre, une demande, un marché, des acteurs - producteurs et consommateurs - qui s'organisent autour d'un contrat et se dotent d'une logistique institutionnelle. L'association est la forme la plus élémentaire de ce contrat pour entreprendre :

- La personnalité morale est acquise par simple dépôt des statuts en préfecture et publication au Journal officiel.

- Il n'existe aucune obligation de déclaration : le Parti communiste est une association non déclarée. 
— L'adhésion et le retrait sont libres et ne sont soumis à aucune formalité.

- Aucun capital minimum n'est exigé.

— La liberté d'organisation est totale.

— Il suffit d'être deux pour créer une association.

À l'inverse du contrat de société qui est un contrat synallagmatique qui doit répondre à certaines exigences (le partage des bénéfices ou des économies, la participation au capital et au comblement des pertes), le contrat d'association n'est soumis à aucun critère particulier et répond à une définition négative : est contrat d'association ce qui n'est pas autre chose, contrat de société commerciale, contrat d'entreprise, etc. Cette définition juridique négative se justifie par l'appartenance du droit d'association au domaine des libertés civiles et individuelles fondamentales. D'abord prohibé par la Révolution, le droit d'association est introduit dans l'ordre juridique par la loi sur les syndicats de 1884, puis par la loi de 1901, pour devenir une liberté constitutionnelle en 1971. Au-delà d'une définition juridique nécessairement succincte, il faut donc rechercher une définition positive de l'association : un outil au service de la mise en œuvre de projets de citoyens exerçant une liberté imprescriptible. Tous les projets ne requerront pas la création d'une association, mais toute association devra être portée par un projet sous peine de n'être qu'une coquille vide. Le projet est un contrat au carrefour de deux tendances contradictoires, la socialisation des moyens à mettre en œuvre (la mise en commun de moyens matériels et financiers) et le désir d'autonomie des individus. La complexité de ce contrat s'accroît avec le progrès social, le besoin d'entreprendre pour le bien commun étant lié à une élévation du niveau d'instruction et d'information, porteur en lui-même de projets individuels et d'affirmation de l'autonomie de la personne.

\section{UN DROIT LONGTEMPS SUSPECT}

$\underline{\text { Retour à la table des matières }}$

Expression des citoyens entre la société civile et l'État, le droit d'association a été longtemps le mal-aimé de la démocratie française. La République s'est construite autour de la théorie élaborée par Jean-Jacques Rousseau dans Le Contrat social qui, pour éviter l'asservissement des plus faibles par les plus forts, prône la fusion des libertés individuelles en une seule: la volonté nationale, désignée par la voie de l'élection et liée au corps social par le contrat social qui a la charge de défendre la liberté de chacun. "Aliénation totale de chaque associé avec tous ses droits à la communauté », la notion de contrat social nie la nécessité de corps intermédiaires ${ }^{1} \mathrm{car}$ « s'il restait quelques droits aux particuliers, comme il n'y aurait aucun supérieur commun qui pût

Tout ce qui permet l'organisation libre des citoyens indépendamment de l'État: associations, syndicats, organisations professionnelles, collectivités locales. 
prononcer entre eux et le public, chacun étant en quelque point son propre juge, prétendrait bientôt l'être pour tous ${ }^{1}$ ». Alexis de Tocqueville a montré comment la Révolution saura reprendre la tradition centralisatrice de l'Ancien Régime et briser tout intermédiaire entre le pouvoir central et les individus. La loi Le Chapelier (1791) interdira toute forme d'organisation professionnelle au nom de la lutte contre les corporations d'Ancien Régime en proclamant que l'anéantissement de toute association professionnelle est une des bases fondamentales de la Constitution française. La théorie de la souveraineté nationale instaurera la condition de citoyen-sujet protégé par l'État auquel il confie la défense de sa liberté par l'exercice périodique de son droit d'élection de la souveraineté nationale. Ainsi s'élabore ce que François Furet appelle « une sorte de droit divin de l'autorité publique qui, une fois élue, n'a plus de comptes à rendre aux citoyens avant l'élection suivante: la Révolution ne dérive vers la démocratie pure que pour mieux assumer, à un autre niveau, la tradition absolutiste ${ }^{2}$ ».

\section{LA CONSTITUTION D'UNE ASSOCIATION}

La constitution d'une association n'est soumise qu'à un minimum de formalités. La déclaration n'est pas obligatoire, mais est nécessaire pour acquérir la personnalité morale. Il faut s'adresser au Bureau des Associations de la préfecture ou de la sous préfecture du siège social de l'association, qui vous fournira tous les documents nécessaires. Le coût est d'environ 250 francs représentant les frais d'insertion au Journal officiel. L'administration ne peut refuser de délivrer le récépissé de déclaration, car il s'agit d'une liberté publique constitutionnelle. Au-delà de ce travail rudimentaire de "paperasserie », l'effort doit se porter sur la rédaction des statuts. Un seul article représente un véritable travail de créativité : l'objet social.

Il doit être licite : toute association portant atteinte à la loi et aux bonnes mœurs, à l'intégrité du territoire national et à la forme républicaine du gouvernement est nulle et pourra être dissoute par l'autorité judiciaire saisie par le ministère public ou par toute personne intéressée. En 1988, des associations dites de "mères porteuses " ont été dissoutes sur saisine préfectorale. L'autorité administrative garde également un pouvoir de dissolution à l'égard des associations :

- qui présentent le caractère de groupes de combat et de milices privées, ou ayant pour but de porter atteinte à la forme républicaine du gouvernement : le service d'action civique (S.A.C.) a été dissous sur ces bases ;

- qui auraient pour but d'exalter la collaboration avec l'ennemi ;

- qui inciteraient à la haine ou à la discrimination raciale ;

- qui se livreraient sur le territoire français ou à partir de la France à des agissements en vue de provoquer des actes de terrorisme.

La dissolution intervient alors par décret en Conseil des ministres.

Il doit être clairement défini et devra correspondre à l'activité effective de l'association.

Le Contrat social, livre I, chap. IV.

François Furet, Penser la Révolution française, Gallimard, 1978. 
L'objet définit le domaine de compétence de l'association : dans toute relation avec les pouvoirs publics (demande d'exonération fiscale, de subvention, d'agrément, etc.) on se référera à l'objet social pour octroyer tel ou tel avantage à l'association.

Autres éléments obligatoires devant figurer dans les statuts: le nom et le siège social. L'association est propriétaire de son nom. Il faudra donc s'assurer de son originalité. Pour le siège social, il faut seulement mentionner la ville, afin de préciser de quelles administrations et juridictions dépend l'association. Ne surtout pas mentionner l'adresse : il faudrait modifier les statuts à chaque fois que l'association déménage !

Sauf si l'on envisage de solliciter la reconnaissance d'utilité publique - qui n'est réellement nécessaire que si l'on désire acquérir un patrimoine étendu, auquel cas il faut adopter des statuts types définis par le Conseil d'État - la rédaction des statuts est libre. Il faudra y édicter des règles concernant la répartition du pouvoir, les ressources et la discipline. De nombreux modèles sont proposés dans les ouvrages spécialisés qui peuvent être adaptés à chaque cas. La recherche de subventions peut nécessiter un agrément ministériel qui imposera l'adoption de clauses types. Il est préférable de s'en tenir aux principes généraux pour les statuts et de renvoyer les règles plus précises de fonctionnement à un règlement intérieur établi et révisable par le seul conseil d'administration.

Sociologiquement, cette absence de corps intermédiaires dans la démocratie française amènera les Français — tant en ce qui concerne les relations des citoyens entre eux qu'avec le pouvoir — à se passionner pour les débats abstraits aux dépens des débats sur la gestion concrète des affaires publiques. Tocqueville avait déjà noté sous l'Ancien Régime comment l'absence de corps intermédiaires et de mandataires entre l'individu et l'État avait amené à transformer les écrivains en hommes politiques. L'imaginaire du pouvoir est préféré à sa pratique effective : « Privés de libertés vraies, les Français vont à la liberté abstraite ; incapables d'expérience collective, sans moyens d'éprouver les limites de l'action, ils s'orientent sans le savoir vers l'illusion de la politique. Faute d'un débat sur la gestion des hommes et des choses, la France est passée à la discussion des fins et des valeurs comme du seul contenu, et du seul fondement de l'activité publique ${ }^{1}$. »

Le droit d'association, comme moyen d'expérimentation sociale et de prise du pouvoir par les citoyens dans la vie quotidienne, s'est trouvé et est toujours handicapé par un tel contexte. L'évolution du cadre juridique est néanmoins favorable. Par une décision du 16 juillet 1971, le Conseil constitutionnel a rangé le droit d'association parmi les « principes fondamentaux reconnus par les lois de la République » de nature constitutionnelle. Cette décision, véritable achèvement de l'État de droit républicain commencé avec la Déclaration des Droits de l'homme et du citoyen du 26 août 1789, a sanctionné le droit à l'intrusion des citoyens dans leurs propres intérêts concurremment à l'État. La loi du 22 juillet 1987 sur le mécénat leur en fournit les moyens en leur

1 Ibid. 
permettant d'affecter une part de leur contribution fiscale à cette entreprise d'intérêt général qu'est l'association.

\section{UNE BOMBE À RETARDEMENT}

\section{$\underline{\text { Retour à la table des matières }}$}

Il est courant d'entendre des lamentations sur le déclin de la vie associative en France, la montée de l'individualisme, le déclin des valeurs collectives, et de conclure que la perspective est sombre pour l'association. C'est là commettre une grosse erreur qui provient d'une cécité devant l'évolution historique.

Tout d'abord la vie associative est peu développée en France si on la compare aux pays de démocratie décentralisée comme les États-Unis ou la Belgique, où plus de la moitié des citoyens ont des responsabilités associatives. On ne connaît pas le nombre exact d'associations vivantes car il n'existe nulle obligation d'en déclarer la mort. Disons que sur les 600000 existantes, touchant 32 p. 100 de la population, 150 à 200000 ont une activité réelle, et qu'il s'en crée autour de 50000 nouvelles par an ${ }^{1}$. Retirons-en le bloc des associations sportives ${ }^{2}$ dont les adhérents ne le sont bien souvent que pour obtenir licence et assurance, le secteur associatif pur ne concerne qu'une partie très réduite des citoyens.

Ensuite, il est vrai que certaines associations connaissent des difficultés, mais un tel constat concerne surtout celles du premier âge de la vie associative, généralement de très grandes associations qui, tels les mammouths en leur temps mourant faute de s'adapter au réchauffement de la planète, ne s'adaptent pas à la nouvelle conjoncture. Ce premier âge a correspondu à l'économie de production de masse où, pour fuir l'anonymat des grandes structures, l'individu cherchait à se regrouper pour organiser sa défense et conquérir des espaces nouveaux de libertés: loisirs, culture, santé... Ce premier âge associatif a voisiné avec l'essor du syndicalisme, tantôt en le précédant — les associations de fonctionnaires ont été la seule forme d'organisation qui leur ait été ouverte avant la reconnaissance du droit syndical en 1945 — , tantôt en en étant le

1 D'après le CICOS (Centre d'information et de communication sociale - Île-de-France) il s'est créé en Île-de-France 10302 associations en 1988 et 11700 en 1989, soit une augmentation de plus de 10 p. 100. 27000 associations d'Île-de-France sont employeurs, regroupant 207000 salariés.

2 La loi de 1986 sur les associations sportives scolaires impose la création d'une association sportive par établissement du second degré, ce qui explique en majeure partie la croissance de la création d'associations depuis cette date. 
prolongement comme le tourisme social qui s'est bâti avec les fonds des comités d'entreprise.

Cette forme d'association traverse la même crise que toutes les organisations représentatives. La société change, l'association aussi.

La société dans laquelle nous entrons - celle du $\mathrm{XXI}^{\mathrm{e}}$ siècle, qui se met en place avec le retour à la prospérité économique de la fin des années 80 — ne sera plus fondée sur l'individu atomisé, écrasé par la machine (immortalisé par Chaplin dans Les Temps modernes), exploité, voyant son intérêt moral, matériel, sa santé, son développement personnel irrémédiablement opposés à la prospérité de l'économie, et ce dans les sociétés capitalistes comme, finalement encore plus, dans les sociétés socialistes.

L'individu ignorant et isolé décrit par Karl Marx au XIX ${ }^{\mathrm{e}}$ siècle fait place à l'individu savant et coopérant d'une économie hautement compétitive d'où la classe ouvrière aura quasi disparu. Cette société du savoir que décrivent Peter Drucker ${ }^{1}$ et Alvin Toffler ${ }^{2}$ : haut niveau d'éducation des élites, dépendance beaucoup plus faible de l'entreprise qui tend à ne devenir qu'un lieu comme un autre d'épanouissement des compétences, possibilité de carrières successives, mais aussi un fossé beaucoup plus grand entre " gagnants » et " perdants », va offrir des possibilités de développement encore jamais vues à la vie associative dont le second âge s'ouvre à nous.

Une demande plus complexe, une offre riche par sa compétence, des effets accrus par le management, la vie associative va changer !

- La demande

1. Le travailleur du savoir sera un gourmand de vie associative. C'est un homme complet, un individu rendu libre parce qu'il sait qu'il transporte son capital le plus précieux avec lui : son savoir. Il choisit son entreprise plus qu'elle ne le choisit. Il sait que ses compétences sont éphémères et que sa véritable force, au-delà de son diplôme qui reste l'incontournable clé d'entrée sur le marché du travail, c'est d'avoir appris à apprendre, c'est de se former pour adapter en permanence son savoir-faire à l'évolution de plus en plus rapide des marchés et des technologies. La compétence devient un enjeu en soi, le travail est surtout un jeu qui le valorise et l'entreprise n'en est plus que le terrain. Ses champs d'intérêt sont donc très divers et il saura aussi bien se consacrer au développement des aciers spéciaux dans les boîtes de vitesses qu'à la gestion d'un club de voile. Il sait que son efficacité n'est pas seulement due à ses performances personnelles, mais surtout à sa capacité à travailler en équipe, à organiser son propre travail, à évaluer et gérer son apport à la société : il

Peter Drucker, Les Nouvelles Réalités - De l'État providence à la société du savoir, Interéditions 1990.

2 Alvin Toffler, Les Nouveaux Pouvoirs, Fayard, 1991. 
construit sa vie plus qu'il ne la gagne et sait faire servir l'organisation à la réalisation de ses ambitions.

L'association peut être pour lui un nouveau terrain d'expérience et d'innovation dans lequel il puisera idées nouvelles et régénération personnelle. La richesse du tissu associatif deviendra un critère du choix de sa résidence comme la présence d'une école, d'un centre culturel ou d'un équipement de loisirs.

2. Les exclus de la société du savoir, qu'ils soient insuffisamment qualifiés ou qu'ils soient en dehors du marché du travail par cumul des handicaps, constitueront paradoxalement l'autre pan de la demande associative. On trouve dans cette catégorie des représentants de l'ancienne classe ouvrière en déclin qui n'aura pas pris à temps le chemin de la reconversion comme les exclus du quart-monde dont l'intégration est tributaire de dispositifs spécifiques que les grands systèmes publics se révèlent incapables de produire. L'héritier de la grande industrie qui sera resté sur un savoir parcellaire et figé, qui n'aura pas appris à apprendre, s'exclut par lui-même du mouvement de la société et des métiers. Il s'affirme de plus en plus un seuil minimum de savoir en deçà duquel se produit le décrochement social et professionnel. Le progrès d'une économie fondée sur les technologies de pointe est tributaire d'une politique de développement des ressources humaines qui sera la seule voie désormais ouverte à la mobilité sociale. Le $\mathrm{XXI}^{\mathrm{e}}$ siècle rejettera le self-made man sans diplôme parvenu au seul prix de son énergie. L'obstacle de la formation est incontournable: aux organisations professionnelles, à la société civile de rechercher les modalités de formation adaptées aux spécificités de chaque culture ou de chaque handicap.

3. La compétitivité d'une économie n'est plus aujourd'hui seulement celle de ses entreprises, mais la compétitivité globale de son système social. Entre la France qui gagne et la France qui perd, il n'est pas envisageable de laisser s'élargir un précipice qui serait la tombe de la démocratie. Une société ne se nourrit pas seulement de résultats d'entreprises performantes mais des valeurs qui la soudent, lui donnent ses ambitions et sa raison de vivre. Nous sommes entrés dans un monde de compétition, de progrès et d'évolution continue des marchés, d'obsolescence rapide des outils de production et des connaissances. L'ouverture des frontières, l'internationalisation de l'économie imposent une capacité d'adaptation au changement permanent, au dialogue entre les cultures, soumettent la société à des tensions accrues. Le changement va aller de plus en plus vite et la société aura de plus en plus besoin de cohésion. La vie associative peut être ce lieu d'apprentissage de nouvelles formes de coopération, de création de nouvelles valeurs collectives et d'art de vivre en société, qui sont la clé de la compétitivité de l'entreprise France. 
Le reaganisme aux États-Unis a sonné le glas des idéologies qui tout au long du $\mathrm{XX}^{\mathrm{e}}$ siècle ont entendu plier le corps social à leurs dogmes en opposant tour à tour le politique, l'économique ou le social : l'un ne pouvait gagner que s'il soumettait l'autre ${ }^{1}$. Le XXI $\mathrm{XX}^{\mathrm{e}}$ siècle sera celui de la synthèse : la compétitivité économique sera inenvisageable sans cohésion sociale. Aucun grand mythe politique ne verra plus le jour sans une capacité à entrer en symbiose avec la société civile. Un rapport établi par l'université de Columbia ${ }^{2}$ dresse le bilan des années Reagan : ruine du système éducatif, du système de santé, défaillance de tous les systèmes de régulation publics entraînant une inflation des litiges et des procédures, une pauvreté structurelle renforcée par la drogue et la criminalité. Pour les auteurs, l'issue ne peut être que dans une complémentarité de l'intérêt public et de l'intérêt privé, de l'économique et du social, et dans un meilleur management de la société américaine dans son ensemble ${ }^{3}$. L'explosion du tiers secteur (l'ensemble des organismes à but non lucratif) aux États-Unis est une première réponse à ce défi.

- L'offre sera assurée par la formidable disponibilité en matière grise de la société du savoir. En économie productiviste, un ouvrier à cinquante ans était épuisé et considéré comme ne pouvant plus que reproduire les tâches initialement apprises. Un cadre se pressait comme un citron et arrivait vidé à l'âge de la retraite. Le savoir devenu le capital principal, il s'entretient et offre de nouvelles possibilités de développement de carrière à tous âges. Les aléas de conjoncture, les défaillances d'entreprise, les nécessaires compressions de coûts de structure mettent précocement en préretraite des éléments parfaitement opérationnels désireux de continuer à développer leurs compétences dans ce nouveau domaine qu'est pour eux l'intérêt général. Les exemples abondent aujourd'hui de préretraités de 55 ans qui se lancent dans la création d'une entreprise ou dans une nouvelle carrière. D'autres plus soucieux de donner un sens social à leur existence s'engagent dans le mouvement associatif. Le mouvement EGEE (Entente entre les générations pour l'économie et l'emploi) s'est ainsi développé à partir de cadres préretraités et a permis de mailler le territoire d'un réseau de soutien aux créateurs

Ce qui de toute façon était déjà faux dès le $\mathrm{XIX}^{\mathrm{e}}$ siècle : des patrons philanthropes et visionnaires comme Godin ont démontré que la prospérité économique à long terme et le progrès social allaient de pair.

2 "Global competitiveness: Getting the US back on track », The American Assembly, Université de Columbia, WW Norton N.Y., 1988.

«... Aucune nation ne dépense plus que nous pour le système de santé et l'éducation; nos résultats sont cependant médiocres. Nous dépensons massivement en recherche et développement, mais nous échouons à en commercialiser les résultats. C'est là l'œuvre d'une entropie institutionnelle de l'Amérique qui fait que nous ne faisons pas un usage efficient et efficace de l'argent que nous dépensons. Nous n'avons plus désormais de chèque en blanc sur les divers secteurs de notre économie, et nous devons porter notre attention beaucoup plus sur comment nous dépensons... » Op. cit., p. 20. 
d'entreprises. L'entreprise ne consommera pas toute la matière grise disponible : aux associations d'en profiter !

\section{- Le management}

Aux États-Unis, la fin des années Reagan a vu un progrès considérable de la productivité des organismes à but non lucratif en réaction au déclin de la capacité de régulation des institutions publiques et aux gigantesques problèmes de cohésion sociale qui en résultent. À volume constant de dons volontaires, beaucoup d'organisations arrivent à un bien meilleur résultat. Les Girls Scouts ont ainsi pris en compte les aspirations des femmes à une vie professionnelle et ont pu s'ouvrir aux femmes des minorités de couleur en leur offrant les services jusque-là réservés à la majorité blanche. Les Girls Scouts of USA » ont réussi à garder leurs 3,5 millions d'adhérents bien que la population d'âge scolaire ait baissé de 20 p. 100 de 1978 à 1988 : elles ont donc sensiblement accru leur part de marché ${ }^{1}$.

En France, l'aide humanitaire a vu se mettre sur pied en une dizaine d'années des organisations ultra-performantes capables de monter une opération internationale en réaction directe à l'événement.

En matière d'aide au développement, la contribution des ONG est croissante : En 1983, les ONG des pays industrialisés ont accordé aux pays en voie de développement environ 3,6 milliards de dollars de dons (soit 13 p. 100 du montant de l'aide officielle), la plus grande partie de ces dons étant collectée par leurs soins. En 1987, elles fournissaient quelque 5,5 milliards de dollars - soit près de 1 milliard de plus que les crédits de l'association internationale pour le développement ${ }^{2}$.

À l'approche caritative se substitue l'approche managériale qui invite chaque dirigeant à se poser la question de la rentabilité sociale de ses actions : quel est mon objectif, comment et avec quel moyen puis-je l'atteindre, quel niveau de performance puis-je escompter, les moyens mobilisés (collecte de dons, bénévolat) sont-ils en rapport avec les résultats obtenus? Le management permet de définir une vision stratégique autour de laquelle se définit la cohérence de l'organisation, la mission et le niveau de performance de chaque responsable, et de mieux gérer le bénévolat qui est, moins que jamais, un droit à l'incompétence. À la notion de bénévole se substitue celle de professionnel non rémunéré.

Plus complexe par sa nature même que l'entreprise, l'association devient le terrain des pionniers et des innovateurs, et anticipe le management de demain.

Peter Drucker, op. cit., p. 231.

Finances et Développement, revue du FMI et de la Banque mondiale, décembre 1990. Cette contribution a représenté 14 p. 100 de l'aide totale en 1987, dont 3,3 milliards de dollars de collecte propre. 
Aux États-Unis, le secteur non lucratif - qui est le premier employeur avec l'équivalent de dix millions d'emplois à plein temps — devient une véritable école de management pour le monde des affaires. Peter Drucker ${ }^{1}$ souligne qu'elles savent faire ce que ne savent pas faire les entreprises, empoisonnées par le dogme du profit à court terme : Les OBNL (organismes à but non lucratif) partent de l'environnement, de la communauté, du client; elles ne partent pas, comme on a tendance à le faire dans les sociétés américaines, de l'intérieur, soit de l'organisation et de la rentabilité financière. $\mathrm{Au}$ sein de ce secteur se forme un management qui choisit ses indicateurs de performance en dehors de l'organisation, qui sait définir des objectifs à moyen terme: le nombre d'alcooliques, de délinquants récidivistes diminue-t-il ? L'Armée du salut est devenue un modèle d'organisation où une équipe exécutive est responsable de l'accomplissement d'objectifs clairement définis par un bureau directorial. Le tiers secteur devient le lieu d'apprentissage du dialogue entre l'économie du quotidien et les objectifs immatériels des missions sociales des entreprises. L'entreprise citoyenne de demain se construit au travers des expériences de management menées dans les organisations de scouts!

\section{UNE ENTREPRISE DE COHÉSION SOCIALE}

\section{$\underline{\text { Retour à la table des matières }}$}

La société est un train dont la locomotive sera de plus en plus moderne et ira de plus en plus vite car elle sera l'objet de toutes les sollicitudes. Cela est normal et nécessaire car le TGV d'aujourd'hui est le tortillard de demain. Mais on ne roulera pas à cinq cents kilomètres à l'heure avec un wagon de queue brinquebalant. Si tous les pays sont à terme capables de construire la locomotive, on jugera de leur compétitivité à la capacité à assurer la cohésion du train : l'U.R.S.S. a su fabriquer des fusées interplanétaires mais n'a pas su ravitailler les boulangeries. Il est paradoxal que la France soit traversée par des tentations totalitaires qui remettent en cause les fondements mêmes de la tradition républicaine alors que notre économie dégage des pôles de compétitivité qui la mettent dans le peloton de tête des nations industrielles. Le $\mathrm{XXI}^{\mathrm{e}}$ siècle avait vu se développer de tels mouvements en périodes de crise, crise économique et crise des valeurs unificatrices de la Nation allant de pair. $\mathrm{Au} \mathrm{XXI}{ }^{\mathrm{e}}$ siècle tout change : la compétitivité est fonction de la stabilité sociale qui seule permet une reproduction harmonieuse et performante de ce capital essentiel qu'est le savoir.

Peter Drucker, «What business can learn from nonprofits », Harvard Business Review, juillet-août 1989. 
Le progrès reste porteur de déstabilisation sociale avec son cortège de tentations totalitaires. Ainsi, Alvin Toffler nous dit-il :

« [...] Le Dr William Tafoya, un des grands experts du FBI en matière de prévision, assure que dans les dix prochaines années, l'aggravation du malaise social s'accompagnera d'une prolifération de groupes inspirés par la haine [...]. Dénonçant le chômage, la misère, la situation des sans-abri et l'analphabétisme comme les grandes sources du malaise social, Tafoya a calculé la fréquence croissante des crimes, émeutes et agressions liés au racisme ; il en conclut que l'encadrement destiné à assurer la justice sociale n'est plus actuellement que "paille sèche", et qu'il suffirait d'une étincelle pour y mettre le feu [...]. Restant attachés à une conception dépassée du progrès, beaucoup d'Occidentaux présupposent que les idéologies fanatiques, irrationnelles et fauteuses de haine, disparaîtront de la surface de la terre à mesure que les sociétés deviendront plus "civilisées" [...]. L'émergence d'une économie neuve et telle que le monde n'en a jamais connu, qui exige de rapides changements dans les modes de travail, les styles de vie et les habitudes, paraît redoutable à beaucoup. Terrifiées par l'avenir, des populations importantes cherchent leur salut dans les spasmes et les excès d'une réaction sans compromis. La mutation ouvre des failles par où les fanatiques se précipitent, elle donne des armes à toutes ces minorités dangereuses qui ne vivent que pour la crise, dans l'espoir qu'elle leur permettra de s'élancer sur la scène nationale ou mondiale — et de nous ramener tous aux noires périodes du Moyen Âge ${ }^{1}$. "

Tout comme une entreprise, une nation doit se développer sur trois axes. Cette règle n'a rien de nouveau puisqu'elle a été définie il y a quinze siècles par saint Benoît qui soulignait que le progrès d'une communauté était tributaire d'un cadre physique - le monastère - porteur de son identité et assurant sa subsistance économique, d'un abbé qui en est le chef et a la mission de la guider vers l'accomplissement de son projet, et d'une règle - les choses qui se font et ne se font pas - porteuse du sens de la vie de la communauté, qui régit les rapports des membres entre eux, organise sa cohésion, lui permettant par là d'atteindre les objectifs spirituels et matériels qui la sous-tendent.

La France a aujourd'hui restauré les murs : le monastère est en bon état, les entreprises tournent bien.

Mais les institutions publiques ne sont plus capables de délivrer de grands messages unissant les citoyens comme a pu le faire l'école de la III $^{\mathrm{e}}$ République autour de la mythologie républicaine. Liberté, égalité, fraternité n'ont sans doute jamais été aussi nécessaires ni actuels, mais c'est le management public qui pèche et n'est plus capable de souder les citoyens par une vision unificatrice. La société française manque de projet et il y a danger

Alvin Toffler, op. cit., p. 305-307. 
car le ventre est encore fécond qui engendra la bête immonde ${ }^{1}$ : il faut donner du sens à notre performance!

Quant à la règle du jeu, bien peu de choses ont changé depuis que le général de Gaulle a décrit à Bayeux en 1946 la propension de notre vieux peuple gaulois aux querelles intestines. Nous devons apprendre à travailler ensemble autour d'un projet ambitieux qui donne du sens à notre vie, à confronter nos points de vue différents comme autant de richesses contribuant au bien commun et non comme des prétextes à des guerres de religion destructrices.

Le Japon au sortir de la guerre était ruiné, l'empereur incarnant l'unité et la pérennité de la nation n'échappa au procès pour crime de guerre que par la bienveillance du vainqueur. Mais le pays gardait sa cohésion et il a su en faire le capital qui lui a permis de progresser sur l'axe économique.

Chaque économie s'appréciera de plus en plus par son point faible: les mouroirs de Calcutta font oublier que l'Inde pourrait être une grande puissance industrielle. Le quart-monde croissant au cœur de New York éclipse les Boeing de Seattle, et les exclus de la prospérité sont une épine dans le pied de la France qui gagne.

\section{Le monastère : un modèle de management pour l'association?}

L'observation de la vie d'un monastère bénédictin illustre la plupart des principes de management qui seront développés dans cet ouvrage :

- La communauté se regroupe autour d'un projet précis : le combat spirituel vers une vue optimiste de l'homme transcendé par Dieu.

- Elle a des valeurs, par définition éternelles, puisque ce sont celles de l'Évangile.

- Elle a un manager, le père abbé, qui est le garant des objectifs de la communauté et de son style de vie. Il a une double mission : assurer la cohésion de la communauté et sa bonne gestion matérielle afin de dégager l'individu des contraintes et lui permettre de se consacrer à son projet spirituel, et soutenir chaque membre dans son projet personnel pour qu'il trouve sa voie propre dans le projet commun.

- Elle est régie par une règle qui est un soutien pour celui qui s'engage dans le projet monastique. Elle définit les compétences de l'abbé, en limitant ses pouvoirs à l'application stricte de la loi divine pour éviter tout pouvoir personnel, et établit les valeurs partagées et la loi commune.

- Elle a une identité forte : un lieu chargé de symboles, chaque monastère ayant son histoire et sa spécificité.

- Elle est économiquement performante, l'activité lucrative est un moyen de poursuite du projet en libérant les moines des contraintes temporelles et s'appuie sur une stratégie marketing efficace :

très forte différenciation des produits, qualité et image de marque. Les monastères sont aujourd'hui des PME très bien gérées.

- Elle pratique le management de la qualité : veillant beaucoup à ce que l'institution, la

1 Bertolt Brecht parlant de l'actualité du retour du fascisme et du nazisme. 
structure, soit au service de la personne [...] pour éviter que la personne ne soit d'abord au service de la structure ${ }^{1}$. Son organisation est centrée sur la réalisation du projet : saint Benoit soulève tout cela jusqu'au monde spirituel, en arrachant à la terre ce qui est de ta terre et en lui donnant le sceau de la consécration divine ${ }^{2}$. L'organisation est souple : elle a peu de niveaux hiérarchiques (entre trois et cinq, alors que les entreprises performantes arrivent difficilement à descendre au-dessous de dix) et pratique la rotation de chacun dans les fonctions, contribuant à abattre les cloisonnements.

- Elle a une communication active... tout en ne disant rien : les moines ne parlent pas mais on parle d'eux, ils sont accueillants, participent à l'animation de la cité, animent un réseau de leaders d'opinions dans le monde culturel et spirituel.

Ce modèle du genre est-il transposable ? À chacun d'y méditer... mais ça fait quinze siècles que ça marche!

Une société, comme tout système, ne vaut en fin de compte que ce que vaut le plus faible de ses éléments : c'est par la manière de traiter ses pauvres, ses illettrés, ses malades, de faire progresser le niveau général d'éducation et non pas seule ment celui des élites ${ }^{3}$ que l'on distinguera les sociétés compétitives de celles qui ne le sont pas.

C'est désormais le social qui entraîne l'économique, contrairement aux credo économistes et marxisants qui ont imprégné l'intelligentsia du $\mathrm{XX}^{\mathrm{e}}$ siècle. Le plus grand bouleversement de la société française d'après-guerre, la modernisation de l'agriculture, est venu de l'action d'éducation populaire menée par la JAC (Jeunesse agricole chrétienne). Elle a abouti à la mise en place de structures de coopération à la base de la gestion d'exploitations modernes que nul n'aurait imaginées au sortir de la guerre ${ }^{4}$.

Reconnaissons à Louis XVI cette intuition : en jouant au serrurier n'avait-il pas la perception que le management d'une société est comme une mécanique complexe faite de cinématique, de mécanismes bien huilés, de pièces fermement travaillées, mais que tout peut échouer par défaillance de la pièce la plus vulgaire, la plus grossière, la clé en définitive de l'ouverture, le pêne?

\section{LA COHÉSION DU SYSTÈME SOCIAL : LE MODÈLE TRIDIMENSIONNEL}

1 Entretien avec Dom Philippe Aubin, abbé du Bec-Hellouin, dans Parloir - Une vie régulière au Bec-Hellouin, d'après les entretiens filmés par Jean-Paul Cayeux, Éditions Seghers, p, 92.

2 Id., p. 279.

3 Ce qui ne veut bien évidemment pas dire qu'il faille opposer progrès des élites et action sociale. Il faut penser cohésion et non plus opposition : les élites quitteront les pays dont le système sera déliquescent (cas des pays d'Europe orientale, de l'Afrique et de bien d'autres) et la cohésion d'un système ne peut se faire qu'autour de maillons forts que sont les élites.

4 Comme le souligne Henri Mendras (La Seconde Révolution française, 1965-1984, NRF, Gallimard) : Le changement social deviendra de plus en plus le résultat d'une multitude d'agencements entre les grandes forces technologiques, économiques et politiques mondiales et les stratégies des groupes sociaux de base. » (p. 188.) 
$\underline{\text { Retour à la table des matières }}$

Toute organisation sociale se nourrit à trois sources. Un projet: c'est sa raison d'être. Il doit être suffisamment permanent pour être porteur de l'identité du système. Toute organisation sans projet tend à tourner sur elle-même et à être emportée par son entropie naturelle ${ }^{1}$.

La compétitivité globale d'une société est d'abord celle de son économie : c'est ce qui fait la solidité de la maison et assure sa subsistance. Mais elle a également besoin de valeurs ${ }^{2}$ spirituelles qui définissent ses aspirations, ses ambitions, les objectifs qu'elle peut se fixer et autour desquels se mobiliseront les membres de la communauté, les atouts — on ne sait pas tout faire et il faut chercher à se différencier des autres en faisant ce en quoi on est les meilleurs — et le style, la culture qui nous imprègne : nous ne sommes ni des Japonais ni des Allemands et devons trouver notre voie propre. Les membres de la communauté travaillent ensemble grâce à une règle commune porteuse de ses valeurs et qui assure la formation et la coopération et donne à chacun le moyen de s'épanouir individuellement et socialement. Que cette règle du jeu soit déficiente, qu'elle ne recueille pas l'adhésion de l'ensemble de la communauté, qu'elle ne soit plus porteuse de sens, de valeurs partagées, et celle-ci est menacée d'implosion. Le système est donc l'interaction mutuelle d'éléments matériels et immatériels ${ }^{3}$.

Voir chapitre 5.

La crise du XXe siècle a été celle du conflit entre le progrès de la raison et le déclin des valeurs. « La raison ne peut établir des valeurs. Et non seulement elle ne le peut pas, mais l'idée qu'elle le peut est la plus stupide et la plus pernicieuse des illusions. » (Allan Bloom, L'Âme désarmée ou le déclin de la culture générale, Gallimard, p. 221.) L'enjeu du XXIe siècle sera de réconcilier le progrès scientifique et la création de valeurs, ce que prédisait Malraux en annonçant le retour du religieux.

3 Cette prise en compte de l'élément spirituel en sus de l'économique et du social est à la base de la prospérité japonaise. Sur ce point, voir l'ouvrage de René Robin : La Traque de la qualité : vers un management tridimensionnel, Éditions d'Organisation. 


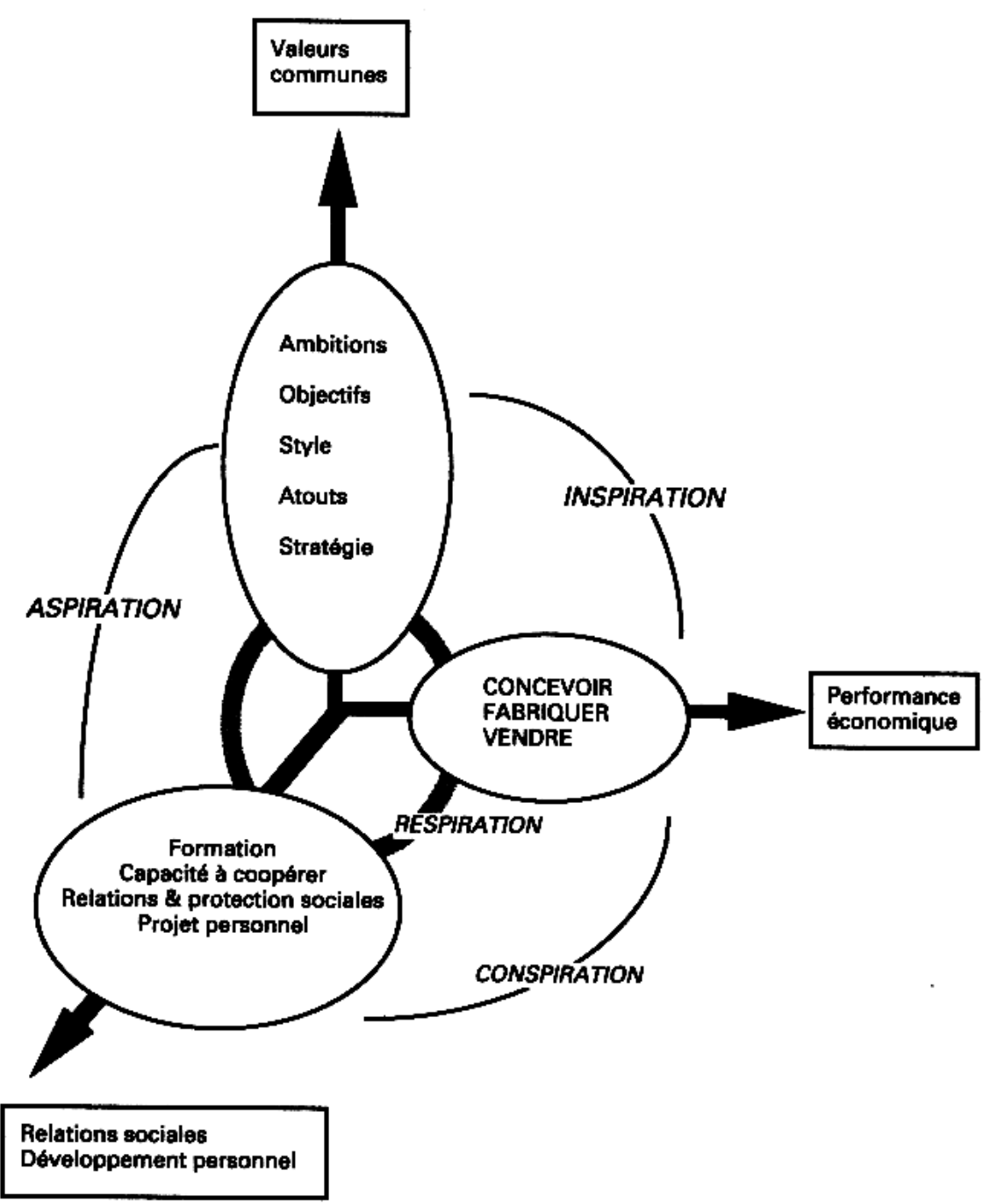

Qui donc a dit que l'association n'avait pas sa part dans ce projet ! Véritable entreprise de cohésion sociale jouant sur les éléments les plus subtils, les plus passionnels et les plus fondamentaux de la nature humaine, dialogue permanent entre le rêve et la réalité, elle requiert le développement d'un management spécifique à la hauteur de ses ambitions. 


\title{
Première partie
}

\author{
L'OUTIL ASSOCIATIF
}

\section{2 \\ Un outil de proposition et d'innovation sociale}

$\underline{\text { Retour à la table des matières }}$

Ғ $\mathrm{n}$ tant qu'outil, l'association permet de créer et de vendre des idées. Une $\amalg$ seule limite est posée par la loi : l'association doit être à but non lucratif, faute de quoi elle devient une société. Contrairement à une opinion encore trop couramment répandue, la non-lucrativité ne signifie pas l'interdiction de faire des bénéfices. Elle est très précisément définie par le code général des impôts sous le vocable de « gestion désintéressée » qui tient en trois principes :

— La non-distribution des bénéfices entre les membres.

- L'absence d'intérêt direct ou indirect des administrateurs dans l'exploitation.

- La non-dévolution ${ }^{1}$ de l'actif aux membres de manière directe ou indirecte en cas de dissolution.

Il peut donc y avoir des associations à but lucratif en toute légalité, elles seront seulement fiscalisées comme une entreprise commerciale.

Cette distinction rigide entre lucrativité et non-lucrativité a été fixée par une jurisprudence de la Cour de cassation (1914 : Caisse rurale de la commune de Manigod) et n'est pas sans présenter des difficultés car les associations ont de plus en plus besoin de développer des activités lucratives accessoires pour financer leur activité principale à but non lucratif, et l'interpénétration est

1 Dévolution : attribution à certaines personnes de biens d'autrui. 
croissante entre marché des idées et marché des produits. Cette confusion est accrue depuis que le Code civil permet au contrat de société de rechercher, outre la répartition des bénéfices, la réalisation d'économies, ce qui peut être également un service poursuivi par une association. Cette distinction n'existe pas outre-Atlantique où les associations ne sont qu'une sous-partie particulière du contrat de société qui exclut la recherche de bénéfices distribuables, qu'il s'agisse de la non profit society des États-Unis ou des « corporations à but non lucratif » du Québec.

\section{LUCRATIVITÉ, NON-LUCRATIVITÉ, ACTIVITÉS ÉCONOMIQUES}

La non-lucrativité n'est pas liée au statut d'association. Il peut en toute légalité y avoir des associations à but lucratif, qui seront alors fiscalisées comme des sociétés.

La notion de non-lucrativité est aujourd'hui devenue complexe : une même association peut avoir une activité économique et des activités lucratives accessoires.

$\mathrm{Si}$ on laisse de côté les associations à but exclusivement lucratif qui ne sont que des sociétés déguisées, et qui n'ont aucun rapport avec l'esprit de la loi du $1^{\mathrm{er}}$ juillet 1901, on peut classer les associations en deux grandes catégories :

\section{Les associations sans but lucratif dont la gestion est désintéressée}

Elles peuvent prétendre à certains privilèges fiscaux si elles répondent cumulativement aux cinq critères fixés par la jurisprudence du Conseil d'État ; (Croix-Rouge française, 31 décembre 1957) et qui sont la définition de référence de la gestion à but non lucratif :

- L'association doit présenter une utilité sociale et viser des besoins qui ne sont pas normalement pris en compte par le marché. Ainsi l'association ne peut être utilisée pour exercer une concurrence déloyale contre une entreprise du secteur commercial. À défaut elle se voit appliquer le même régime fiscal.

- La gestion de l'association ne doit procurer aucun profit direct ou indirect à ses fondateurs ou dirigeants ou membres. Le profit indirect peut consister en avantages en nature ou en la possibilité de répartir l'actif entre les membres en cas de dissolution.

- L'activité exercée doit entrer strictement dans le cadre de l'activité générale désintéressée de l'association et y contribuer par sa nature même, et non simplement financièrement. Une association à but non lucratif ne pourra donc ouvrir une épicerie même si les bénéfices sont réinvestis dans l'œuvre associative. Une même association peut poursuivre simultanément une activité intéressée et une activité désintéressée, mais elle se verra appliquer des régimes fiscaux différents selon la nature de l'activité. En tout état de cause, la recherche de bénéfices est compatible avec le désintéressement mais ne doit être ni systématique ni l'objet premier de l'association.

- La réalisation d'excédents de recettes ne doit pas être systématiquement recherchée.

- Lorsqu'ils existent, ces excédents doivent être réinvestis dans l'œuvre elle-même. La qualification d'association à but non lucratif ne résulte donc pas de la déclaration de l'œuvre conformément aux dispositions de la loi du $1^{\mathrm{er}}$ juillet 1901 ni de la mention dans les statuts de l'intention d'œuvrer sans but lucratif, mais de l'examen par l'administration, sous le contrôle du juge administratif, du caractère effectif de son objet et de la manière dont elle le poursuit. Cet examen peut se faire au moment d'une demande d'agrément ministériel ou de la reconnaissance d'utilité publique, ou par les services fiscaux lorsqu'ils sont amenés à vérifier la légalité des exonérations fiscales consenties aux associations à gestion désintéressée.

\section{Les associations exerçant une activité économique}


Cette distinction est utilisée pour l'application de la législation sur les faillites aux associations

(loi du 25 janvier 1985) ou pour lui permettre d'émettre des titres associatifs (loi du 11 juillet 1985.)

Une association ayant une activité économique est une association dont le but statutaire implique nécessairement l'accomplissement d'une activité de «production, de transformation ou de distribution de biens meubles ou immeubles, de toutes prestations de service en matière artisanale, industrielle ou agricole » (réponse du garde des Sceaux, Journal officiel du 6 décembre 1983). Une activité économique est compatible avec une gestion désintéressée, mais est assujettie à la TVA, sauf dans certains cas limitativement énumérés par la loi.

La confusion entre le partage des bénéfices entre les membres (seul prohibé par la loi de 1901) et la réalisation de bénéfices est couramment répandue. Même sous statut associatif, la réalisation de bénéfices reste la condition de la poursuite de l'activité de l'association. L'évolution de la jurisprudence judiciaire et administrative est constante en ce domaine et lève toute ambiguïté : «Une association est en droit d'accomplir de façon répétée des actes onéreux dans une intention spéculative, dès lors que ceci est conforme au caractère désintéressé de l'objet social et nécessaire à la poursuite d'une activité dont il ne constitue que l'accessoire. » (Cour d'appel de Reims, 19 février 1980.) L'association peut accomplir des actes de commerce, mais ne peut avoir la qualité de commerçant ni être inscrite au registre du commerce. Dans les grosses associations ayant d'importantes activités commerciales, il sera donc préférable de les regrouper dans une société filiale qui pourra être inscrite au registre du commerce et bénéficier de tous les avantages afférents, notamment les baux commerciaux.

Le contrat d'association est donc par essence le domaine de l'imagination et de la créativité. Il en est, bien sûr, fait un usage abusif, lié à sa facilité de mise en œuvre : l'administration y fait abondamment recours pour échapper à ses propres règles de gestion, ainsi que les partis politiques lorsqu'ils recherchent une carte de visite plus anonyme et anodine. Ce qui distinguera la véritable association de la fausse, c'est l'existence d'un véritable projet auxquels adhèrent des membres.

\section{UNE ENTREPRISE DE PROJET À VOCATIONS MULTIPLES}

Retour à la table des matières

L'outil associatif est un circuit court de production de biens collectifs. Dans le mode de production administratif, si l'on désire qu'un service collectif soit mis en place, il faudra aller voir les élus, l'administration, qui passera le projet dans la moulinette de procédures lentes, absconses, détestant par-dessus tout l'intrusion de l'usager. Il faudra collecter les impôts nécessaires et décider de les affecter au projet après prélèvement des frais de gestion. Le circuit court 
verra des citoyens se regrouper autour d'un projet, concevoir le produit répondant à la demande, monter la structure de gestion appropriée, y affecter des financements par un impôt optionnel — en partie déductible de l'impôt obligatoire par le biais du mécénat — représenté par les cotisations.

Proche des citoyens, l'association est à même de concevoir des services adaptés à la demande et de les mettre à la disposition des usagers dans de bonnes conditions d'efficacité.

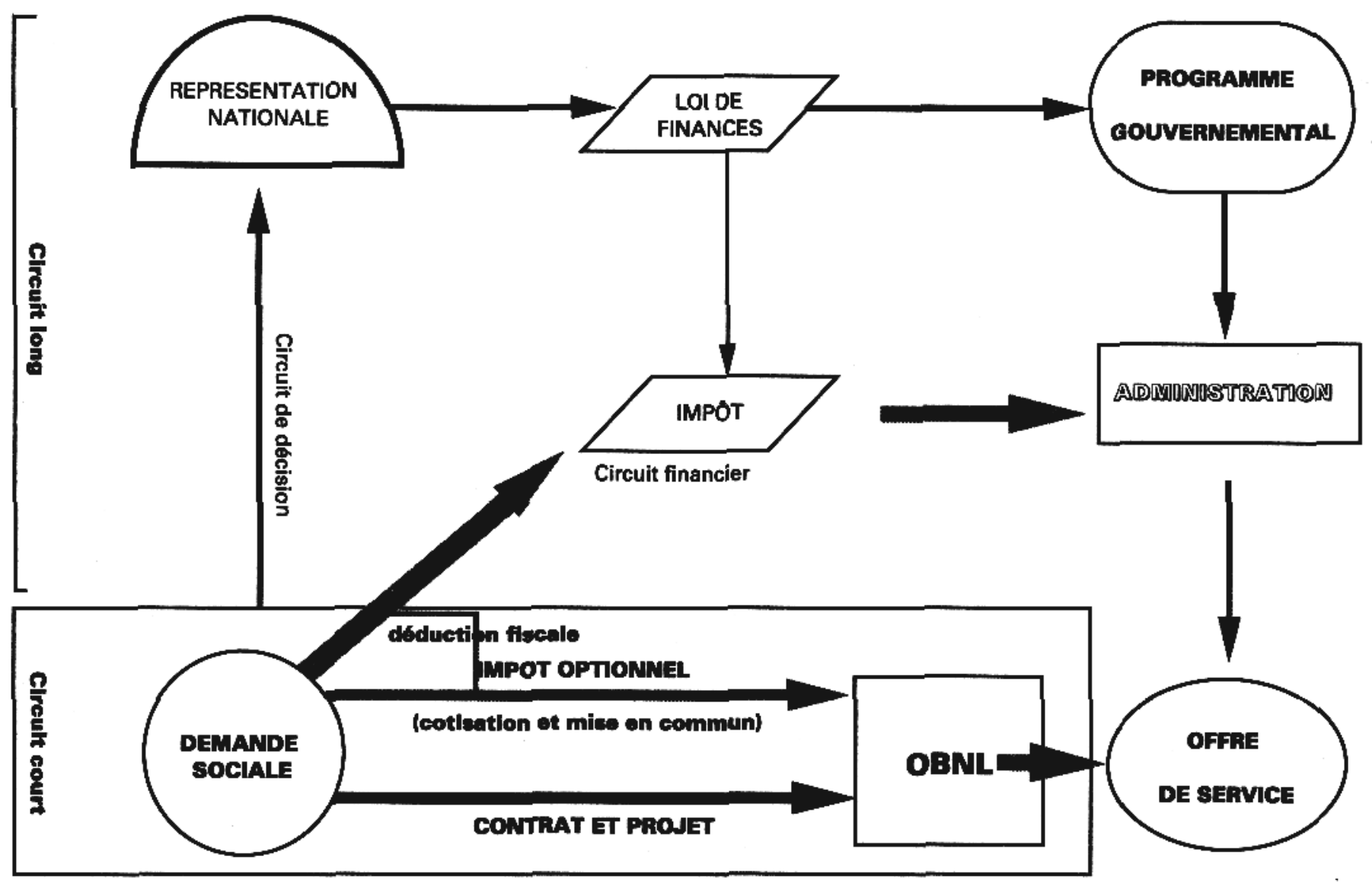

Le mécénat vise à créer un circuit court de production de biens collectifs sans passer par l'administration.

L'aide humanitaire à la grande pauvreté est un service de création presque totalement associatif. Notre système de protection sociale a longtemps été un des meilleurs du monde, mais il repose sur le dogme de la Sécurité sociale dont est aujourd'hui exclue, avec l'apparition d'un fort chômage structurel, toute la catégorie dite des «nouveaux pauvres ». Il est donc, en quelques années, devenu totalement inefficace pour toute une partie des usagers, précisément ceux qui en avaient le plus besoin. Au travers des organisations humanitaires, le circuit court médecin-malades-exclus du système de santé s'est créé, financé 
par la collectivité et par le biais de la collecte de dons. La demande a été identifiée avec précision: cumul des handicaps, très forte imbrication des problèmes de santé et des problèmes sociaux, entraînant rapidement la mise en place de réponses appropriées, par exemple les dispensaires gratuits de Médecins du Monde et de Médecins sans Frontières, où une large place est faite à l'accueil, à l'écoute et à l'accompagnement d'actions d'insertion. Compte tenu des rigidités de gestion du système public, une telle rapidité et une telle précision de réponse n'étaient pas envisageables.

L'État est aujourd'hui le premier à reconnaître qu'en matière de production de biens collectifs, il ne doit pas tout faire, que son fonctionnement est lent, coûteux et qu'au bout du compte le produit fini correspond peu à la demande. Aussi préfère-t-il se dégager de la gestion opérationnelle pour se consacrer à la mise en place des dispositifs d'impulsion qui seront relayés par un partenariat avec les associations permettant une bonne adaptation des politiques au terrain. Le Plan emploi du gouvernement, le Revenu minimum d'insertion, mobilisant des ressources financières que seul l'État peut réaliser, ne peuvent être mis en œuvre avec efficacité que s'il existe une collaboration opérationnelle entre ses services extérieurs et le réseau associatif qui assurera tout le travail à long terme d'accueil, d'écoute, d'analyse et d'accompagnement individualisés qui, seuls, peuvent permettre une insertion sociale et professionnelle effective.

L'association permet la mise en œuvre de projets complexes. L'originalité de l'ingénierie ${ }^{1}$ associative est que le service de base n'est pas toujours payé par son bénéficiaire principal. Il peut comporter plusieurs facettes qui constituent autant de services qu'il faut unir en un projet commun (voir schéma de la page 51).

\section{LE MODE DE PRODUCTION ASSOCIATIF}

$\underline{\text { Retour à la table des matières }}$

L'association est donc une entreprise de service d'intérêt général. Plus complexe par la pluralité de ses clientèles et la séparation des bénéficiaires, elle obéit néanmoins à la règle générale de toute entreprise : il y a d'un côté divers ingrédients que l'on met dans une boîte noire dont il sort un produit, un service rendu. Le management va se fixer pour ambition de réaliser une bonne allocation des moyens à l'objectif poursuivi.

Comme toute entreprise, l'association crée, fabrique et vend. Mais elle le fait d'une manière toute particulière qui fait la spécificité du management associatif :

1 Coordination de tous les éléments d'un projet. 
- Comme une entreprise, une association part de la demande existante. Elle peut n'être que celle de ses membres (l'association de propriétaires ou de locataires) ou viser un public plus vaste. Il y aura alors lieu d'analyser avec précision ses besoins, désirs et comportements pour déterminer les composants de la demande. À ce stade, rien ne différencie l'association d'une entreprise du marché. L'originalité du mode de production associatif est sa capacité à prendre en compte les éléments immatériels non marchands de la demande. Qu'est-ce qui distingue un théâtre d'une association culturelle? Tous les deux ont pour but de produire une pièce pour répondre à la demande d'un public. Mais alors que le théâtre s'assurera que la salle est bien remplie en se situant dans les goûts existants d'un public, l'association tendra à aller au-devant de sa demande, en l'anticipant, soit en faisant connaître un auteur encore méconnu, soit en recherchant de nouveaux lieux de représentations (une cour d'immeuble, une usine, une plage...). La vocation de l'association est de prendre un risque devant lequel tendra à reculer le théâtre face aux contraintes financières. Elle devra pour ce faire trouver un autre client que le spectateur — le bénéficiaire secondaire - pour payer ce risque.

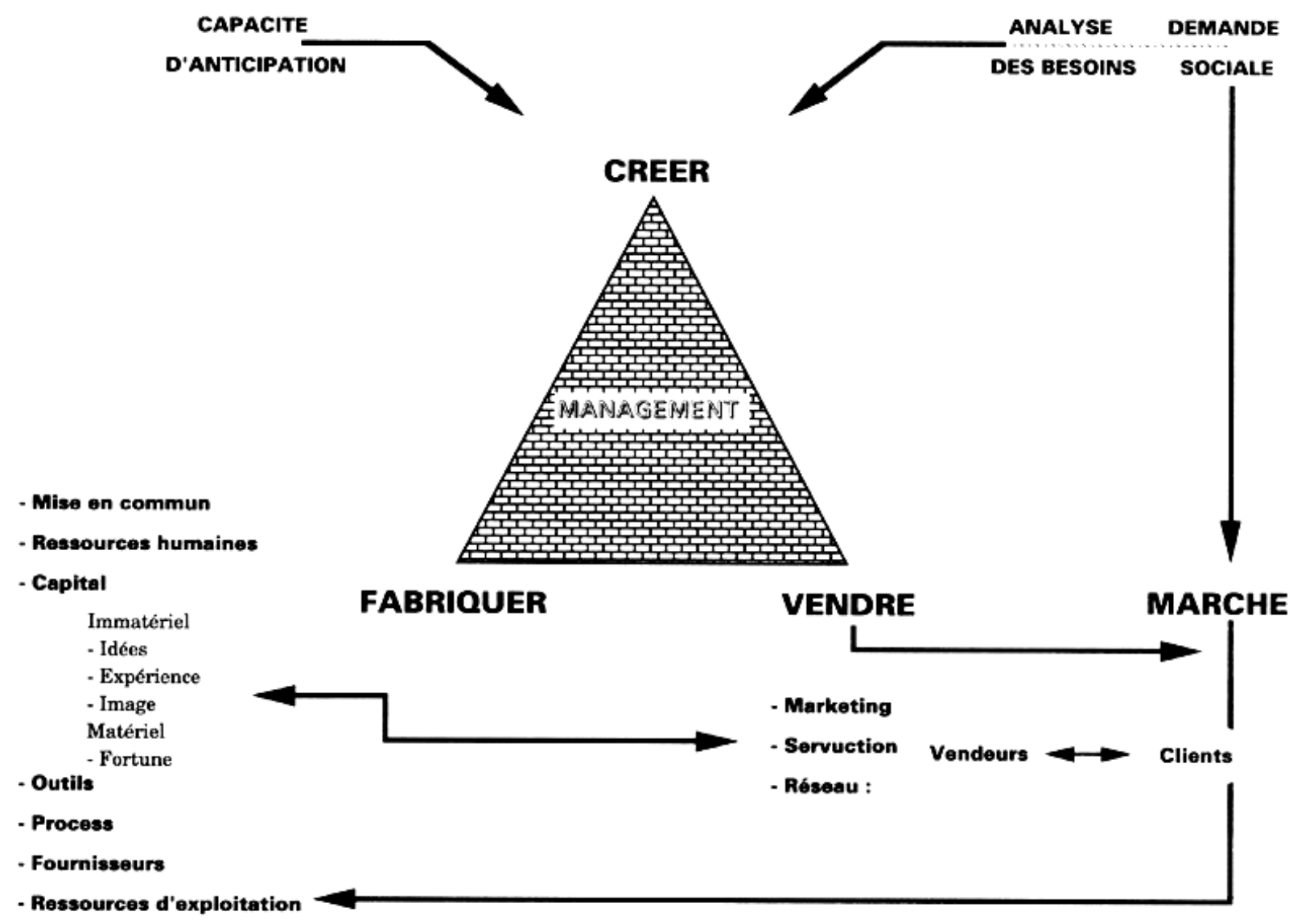


- Tout commence et tout finit avec la demande. Le but de l'association est de vendre son produit à son client principal et de lui apporter un avantage d'une valeur au moins équivalente au prix — en argent ou en effort - payé. Dans l'exemple précité, le public de l'association est celui qui ne serait pas allé au théâtre mais appréciera que le théâtre vienne à lui. L'association aura conçu un ensemble produit (la pièce) et service (un support de représentation sans lequel ce public ne serait jamais allé au théâtre). Le marketing sera l'ensemble des techniques qui permettront l'échange entre ce public et ce couple produit-service. L'objet du marketing est de faire de l'acte de vente (caractérisé par le paiement d'un prix qui pourra être monétaire ou, dans le cas d'une prestation gratuite, un effort, une démarche qui souligne la transaction), qui terrorise tant nombre de responsables associatifs, un acte superflu tant il paraîtra naturel. Car tout aura été centré sur la satisfaction du public, du choix de la pièce au lieu de représentation en incluant l'ensemble des détails matériels qui concourent à la production du service (l’accueil, l'éclairage, la restauration...).

Le marketing est négligé par les associations pour plusieurs raisons :

- Il fait peur, car il touche à un tabou, l'argent, qu'il doit précisément réduire à n'être plus qu'un instrument de mesure de l'adéquation du service aux besoins du public.

- Il nécessite de clarifier les objectifs, de les classer par ordre de priorité pour y affecter les moyens appropriés, débat trop rarement tenu dans les associations.

- Il requiert une pratique réellement professionnelle aux dépens d'un amateurisme blâmable mais encore considéré comme normal s'agissant de bénévoles.

En fait, nous verrons que le marketing est un outil au service des objectifs du projet associatif, et qu'il lui permet de les atteindre sans pour autant perdre son âme, bien au contraire.

Pour fabriquer ce service, l'association dispose de moyens que l'on peut classer par ordre d'importance :

— Le capital : la mise en commun des membres prévue par la loi de 1901 peut s'assimiler à l'apport en capital des actionnaires d'une société. Il est avant tout immatériel : des volontés, du professionnalisme non rémunéré, le réseau de membres et de relations, et se matérialise par le paiement de cotisations. Ce capital s'enrichit avec l'expérience qu'accumule l'association, son histoire, ses essais et ses erreurs, ses succès, l'image qu'elle construit et qu'elle donne d'ellemême, la crédibilité qu'elle se bâtit. Ce capital n'est que secondairement matériel, même lorsque l'association possède un gros patrimoine (immobilier ou mobilier). C'est un piège redoutable et il est difficile de jouer sur les deux registres. Faute de projet et de volonté de satisfaire clients et usagers, la gestion 
et la reproduction du patrimoine prendront le pas sur toutes les autres préoccupations.

- La mécanique de production: c'est avant tout l'art de gérer les ressources humaines, soit la capacité de mettre au service du projet et de la satisfaction du public l'ensemble de la mécanique associative. L'accueil concourt-il à la performance du service au client? La trésorerie est-elle bien gérée ? (Une trésorerie mal gérée génère des frais financiers au lieu de produits et est un impôt de fait supporté par les membres.)

- Les ressources d'exploitation sont le sang de l'association. Une association qui a de grandes idées mais qui ne sait pas mobiliser le premier sou pour les réaliser ne produira rien. Le management associatif sera l'art de réduire les contraintes de gestion du quotidien et du court terme pour permettre le développement du projet à moyen terme, l'art de savoir faire du lucratif pour financer le non-lucratif.

La mise en place de la mécanique associative va donc s'articuler de la manière suivante :

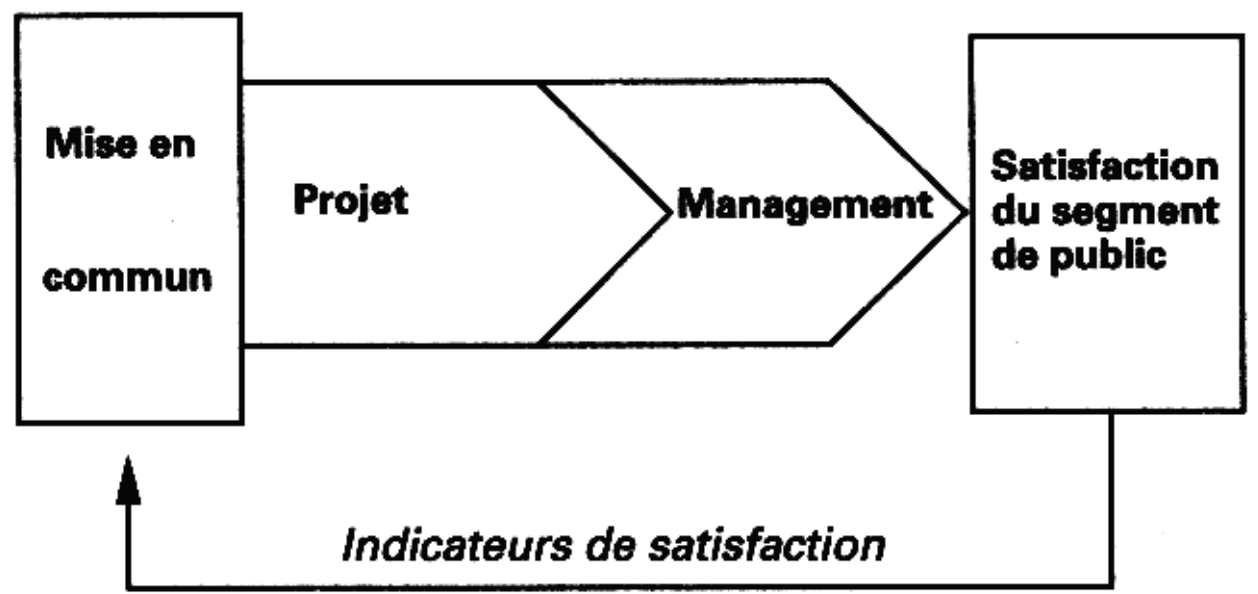

- La première tâche est la conception du projet à partir de la mise en commun de membres, comme réponse à une demande d'un public identifié qu'il s'agira de formaliser, souvent d'anticiper, et de satisfaire.

- La seconde tâche est le management, qui assurera la maîtrise des techniques et des moyens pour que le projet touche son client. Cette organisation de la transaction entre l'offre et la demande est le domaine du marketing qui permet de réduire l'incertitude qui environne l'association par la connaissance des clients, fournisseurs et partenaires de l'association et la mesure de la valeur produite par l'association. La fabrication du service au meilleur coût et pour la plus grande satisfaction du client est le domaine du management de la qualité qui va porter cette orientation du projet associatif au cœur du système de gestion. 
Le dispositif devra être bouclé par la mise en place d'indicateurs de mesure de la satisfaction du public cible qui seuls — indépendamment des déclarations d'intention, de la langue de bois et des velléités — peuvent dire si l'association travaille pour ce pour quoi elle a été créée et lui donner en permanence les moyens d'actualiser son projet.

Un projet complexe : l'entreprise d'insertion

Depuis 1985, les entreprises dites «d'insertion » offrent aux jeunes en difficulté une possibilité d'insertion par la pratique d'une activité professionnelle dans un milieu approprié qui doit permettre l'embauche du jeune dans une entreprise de droit commun. 

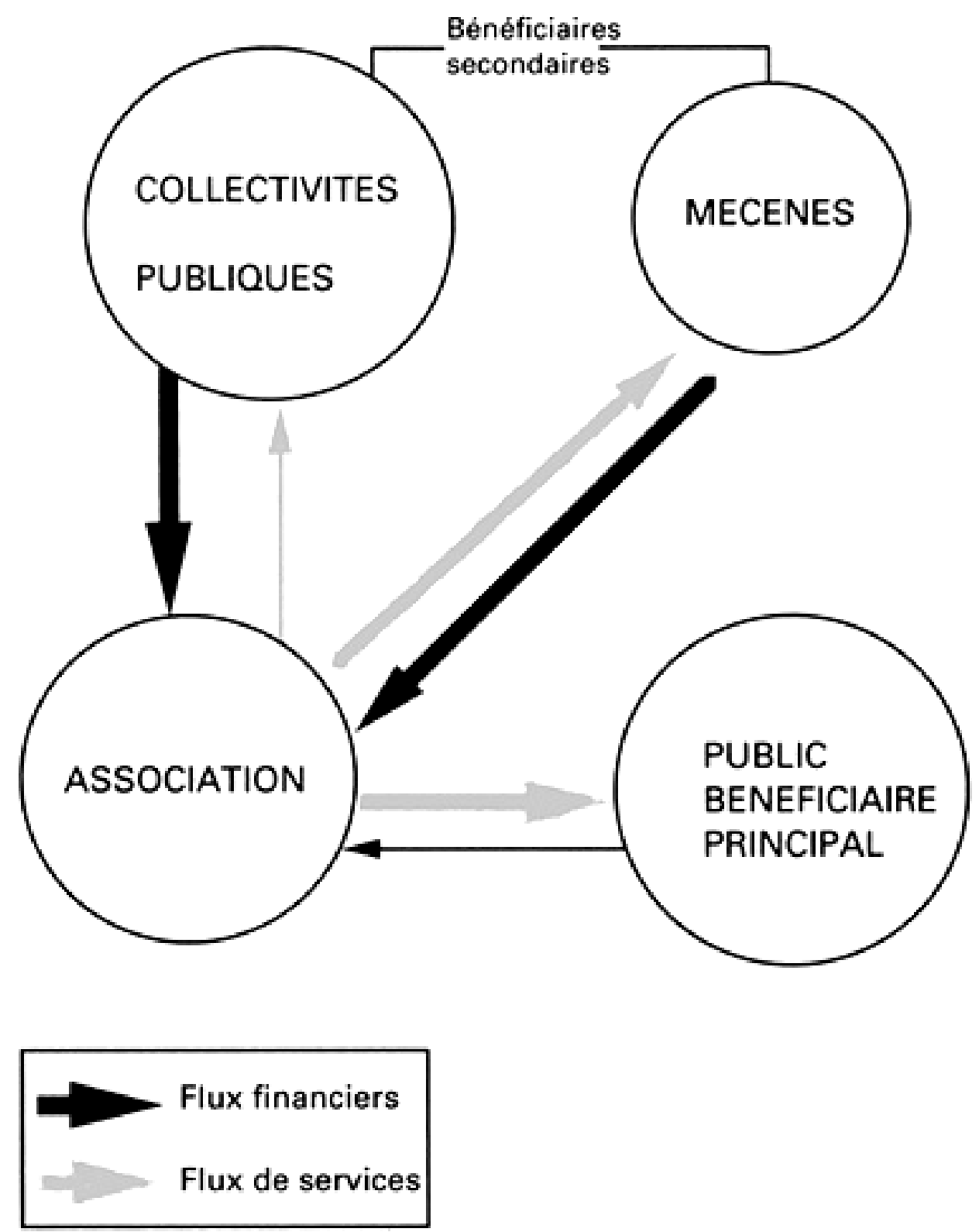

\section{LES RELATIONS DE L'ASSOCIATION AVEC SES PARTENAIRES}

La structure porteuse de projet est complexe en elle-même puisqu'elle comporte :

— une association à l'origine du projet et en charge d'une mission d'intérêt général d'insertion sociale et professionnelle des jeunes ;

- une structure de production de biens marchands qui sera une SARL ou un département à but lucratif de l'association. 
L'entreprise d'insertion (EI) a trois partenaires (voir p. 51 [édition de papier]) :

- Les bénéficiaires principaux sont les jeunes qui trouvent un emploi et une formation adaptée à leurs handicaps sociaux. Ils réalisent une production qui est vendue sur le marché, procurant à l'entreprise des recettes d'exploitation. 


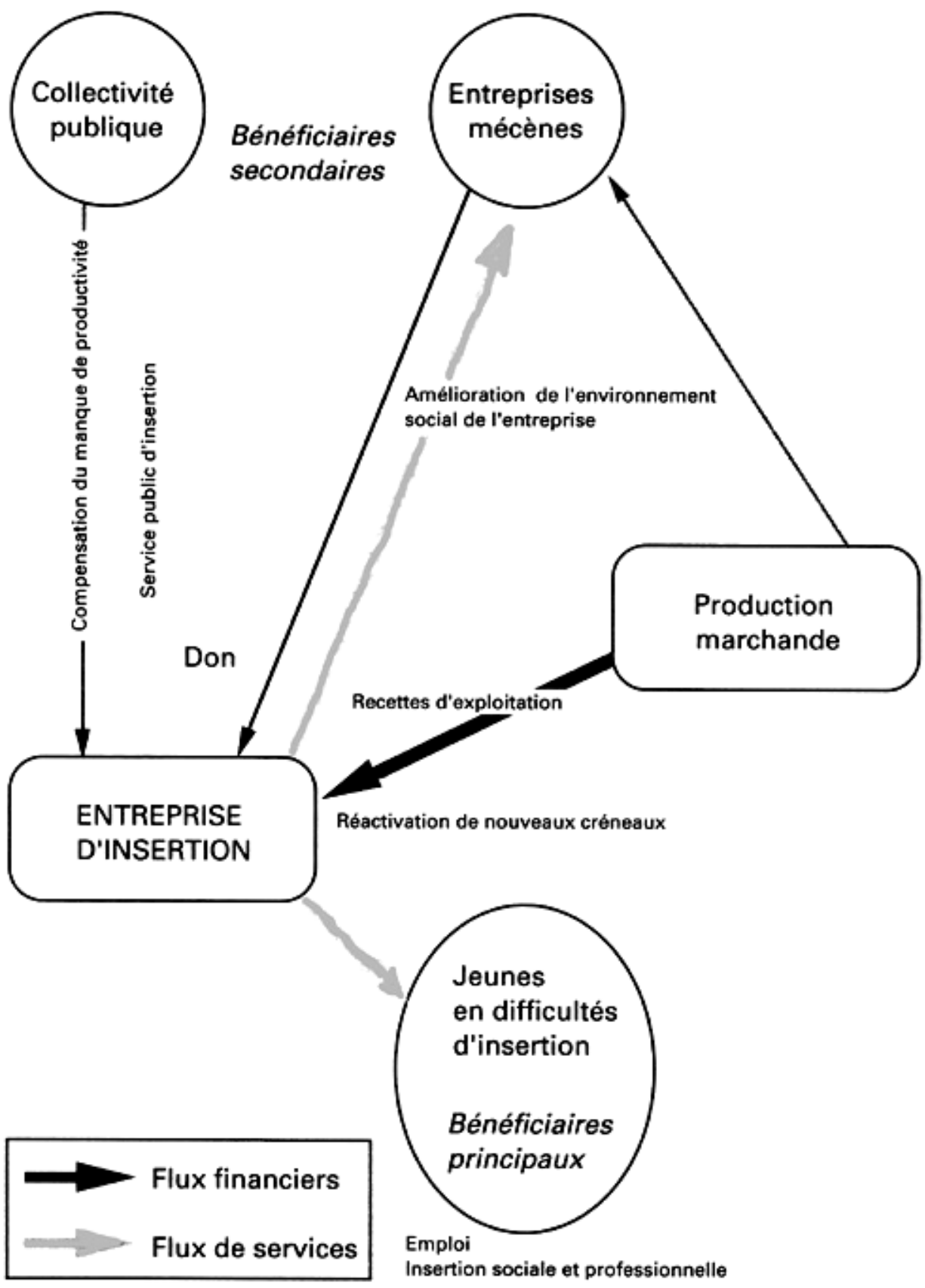

Un exemple de projet complexe : l'entreprise d'insertion

- Deux bénéficiaires secondaires :

- la collectivité publique en charge du service public d'insertion. Un jeune au travail avec une formation, c'est un jeune de moins à la charge de la collectivité. Un jeune en centre d'accueil coûte environ 60000 
francs par an, un jeune délinquant 160000 francs, sans compter le coût des dégâts occasionnés par la délinquance ou la toxicomanie ;

- les entreprises qui ont à faire face à une main-d'œuvre sousqualifiée que ne peuvent gérer les services formation, ou encore à un environnement social dégradé par le sous- emploi et la déqualification. Elles vont trouver dans l'EI un partenaire susceptible de remédier à cette décomposition du tissu social.

Les EI ayant une main-d'œuvre à faible productivité et à faible coût vont pouvoir réactiver certains créneaux de services abandonnés: Darty a ainsi financé des EI pour reconditionner des appareils électroménagers légèrement endommagés et impropres à la vente, activité qui n'est pas rentabilisable avec une main-d'œuvre qualifiée à coût élevé.

Les flux financiers s'organisent de la manière suivante (voir p. 53) :

- Les recettes propres proviennent de la production vendue. Mais elles ne couvriront pas :

- le coût du service rendu sur le lieu de travail ;

- le coût de la sous-productivité de la main-d'œuvre (un toxicomane a une productivité se situant autour de 10 p. 100 de celle d'un travailleur sain).

La recherche de compétitivité de l'EI sera particulièrement délicate à mener car, si elle cherche avant tout la compétitivité, elle aura tendance à recruter les jeunes dont le handicap est le plus léger et, si elle privilégie sa mission sociale, elle risque de s'éloigner de la réalité entrepreneuriale et de retomber dans une logique d'assistance. L'arbitrage devra être permanent et suppose que le manager associatif dispose d'une batterie d'indicateurs reflétant ces deux missions contradictoires et complémentaires.

- Pour permettre à l'EI de fonctionner en condition de sous-productivité, son coût sera imputé à un bénéficiaire secondaire : la collectivité en charge du service public d'insertion. L'État s'y retrouve largement puisque les subventions versées (30 000 francs de subvention forfaitaire auxquels peuvent s'ajouter des subventions spécifiques dans la limite de 60000 francs - circulaire du 31 mars 1989 du ministère du Travail) restent largement inférieures au coût d'un toxicomane ou d'un délinquant et, à terme, sont couvertes par les recettes fiscales générées par l'activité économique de l'EI.

- Le mécène pourra intervenir par deux voies : un apport en capital pour constituer l'entreprise, généralement en passant par une fondation (Fondation de France, Fondation France Active...) qui pourra mobiliser le réseau de compétences nécessaires, ou par achat de denrées.

La crise des finances publiques a amené un renouveau de la créativité associative, la dégageant de la gangue des subventions en créant l'opportunité 
d'un mariage de raison entre la puissance de l'État et la dynamique de gestion de l'entreprise privée que peut représenter l'association. En mettant fin aux rentes sur fonds publics, elle a ouvert l'ère du management, de la remise en cause permanente de l'existant et de la recherche de l'efficacité au service de l'idée.

\section{LE NON-LUCRATIF C'EST RENTABLE ! \\ La création d'une agence de travail temporaire pour les sans domicile fixe (SDF)}

La région parisienne compte 30000 SDF qui trouvent souvent refuge dans le métro et dans les gares. Il s'ensuit une dégradation de la qualité du service public et la SNCF a commandé une étude de faisabilité sur les possibilités de réinsertion des SDF, qui a abouti à la création d'une agence de travail temporaire. Une étude de la population des SDF fait apparaître des tendances lourdes: croissance continue de cette population, et part importante de jeunes sans antécédents sociaux. La majorité de cette population n'est pas installée dans la marginalité, par contre risque de glisser vers une marginalisation structurelle qui rendra la réinsertion beaucoup plus difficile.

Le projet vise donc :

- à stopper le processus de marginalisation ;

- à donner un statut économique aux SDF, ce qui est la clef de l'accès au logement.

Le choix de la réinsertion par l'économique est fait compte tenu de l'expérience des entreprises d'insertion auprès de ce type de population jeune et non qualifiée. L'outil « entreprise de travail temporaire » (ETT) est choisi pour sa souplesse (choix des métiers, possibilité de prendre en compte les handicaps particuliers de la personne, adaptation de l'ETT à la fonction de sas vers un emploi fixe que vise le projet). L'objectif retenu est de réinsérer 250 personnes par an. Le dispositif s'inscrit dans celui des entreprises d'insertion et recevra donc à ce titre une aide de l'État.

Cette opération est-elle socialement rentable ? Qu'en coûte-t-il à la collectivité et quels sont les coûts qui lui sont évités? Patrick Gagnaire, promoteur du projet, en a fait l'évaluation :

\section{Surcoût social de notre action sur une année :}

250 postes d'insertion X 30000 F d'aide par poste 7,5 MF

Coûts évités

RMI 24000 x 250

$6,0 \mathrm{MF}$

Prison (10 p. 100 de la population - $500 \mathrm{~F}$ par jour) 2,7 MF

Hôpital (10 p. 100 de la population - 400 F par jour) $\quad 1,8 \mathrm{MF}$

CHRS (20 p. 100 de la population - $300 \mathrm{~F}$ par jour) 5,4 MF

15,9 MF

La collectivité est donc gagnante de 8,4 millions de francs, et ceci non compris les effets de la délinquance, les torts aux usagers, les dégradations (les SDF sont responsables de 80 p. 100 de celles des gares et des trains), les aides sociales diverses. Il faudrait ajouter le coût de l'effet d'éviction que crée la présence d'une population marginalisée en incitant nombre d'usagers à utiliser leurs véhicules plutôt que les transports en commun. De plus, ajoute Patrick Gagnaire, il faudrait calculer l'effet redistributif de la masse salariale (250 SMIC) dans les flux économiques : «Permettre à un père de famille de trente ans, à la rue, d'avoir un salaire, peut éviter un placement à la DDASS (600 F environ de prix de journée). » 
